\title{
An Adaptive Actuator Failure Compensation Scheme for A Cooperative Manipulator System
}

\author{
A Thesis \\ Presented to \\ the Faculty of the School of Engineering and Applied Science \\ University of Virginia
}

In Partial Fulfillment

of the Requirements for the Degree

Master of Science

in

Electrical Engineering

by

Thummaros Rugthum

September 2013 


\section{APPROVAL SHEET}

The thesis is submitted in partial fulfillment of the

requirements for the degree of

Master of Science (Electrical Engineering)

Thummaros Rugthum, Author

This thesis has been read and approved by the examining committee:

Prof. Gang Tao, Thesis Advisor

Prof. Zongli Lin, Committee Chairman

Prof. Maite Brandt-Pearce

Accepted for the School of Engineering and Applied Science:

Dean, School of Engineering and Applied Science

September 2013 


\section{Abstract}

This research develops a new adaptive actuator failure compensation algorithm for control of a cooperative robotic system subject to uncertain actuator failures. The robotic system has two manipulators to balance a rigid platform, and the right-side manipulator contains one actuator and the left-side manipulator has two actuators (one of which may fail during system operation, but it is uncertain how much, when and which actuator may fail). The developed adaptive actuator failure compensation scheme, based on adaptive integration of three individual failure comepnsators and direct adaptation of controller parameters, is capable of ensuring desired closed-loop stability and asymptotic output tracking, despite the failure uncertainties. A general design procedure is derived, and simulation results verify the desired adaptive failure compensation control performance. 


\section{Contents}

1 Introduction 1

1.1 Literature Review . . . . . . . . . . . . . . . . . . . . . 1

1.2 Organization of Thesis . . . . . . . . . . . . . . . 2

2 Background 5

2.1 Euler-Lagrange Equation . . . . . . . . . . . . . . . . . 5

2.2 Dynamic Model of A Manipulator System . . . . . . . . . . . 8

2.3 Simple Joint Control Scheme . . . . . . . . . . . . . . . . . . . . . . . 11

2.4 Adaptive Control for Robotic System . . . . . . . . . . . . . . . . 12

2.5 Backstepping Nonlinear Control Design . . . . . . . . . . . . . . . . 14

3 Adaptive Actuator Failure Compensation for A Cooperative Manip$\begin{array}{ll}\text { ulator System } & 17\end{array}$

3.1 Problem Formulation . . . . . . . . . . . . . . . . . . . 17

3.1.1 Actuator Redundancy . . . . . . . . . . . . . . 18

3.1.2 Control Objective . . . . . . . . . . . . . . . . . . . 18

3.1.3 Actuator Failure Model . . . . . . . . . . . . . . . . . . . . . . 19

3.2 Derivation of the Dynamic Model . . . . . . . . . . . . . . . . 21

3.2.1 Euler-Lagrange Equation . . . . . . . . . . . . . . . . 21

3.2.2 Determination of Signals $\ddot{\bar{q}}_{1}$ and $\ddot{\bar{q}}_{2} \ldots \ldots \ldots 25$

3.2.3 Dynamic Model in State Space Form . . . . . . . . . . . . . . 27

3.3 Nominal Control Design _. . . . . . . . . . . . . . . . . . . . . . 30 
3.3.1 Backsteping Control Design . . . . . . . . . . . . . . . 30

3.3.2 Nominal Controller Structure . . . . . . . . . . . . . . . 33

3.4 Adaptive Failure Compensation Design . . . . . . . . . . . . . . . 38

3.4.1 Adaptive Controller Structure . . . . . . . . . . . . . . 38

3.4 .2 Error System . . . . . . . . . . . . . . . . 40

3.4.3 Adaptive Laws . . . . . . . . . . . . . . . . . 43

3.4.4 Performance Analysis . . . . . . . . . . . . . . . . . . . 47

3.5 Simulations Study . . . . . . . . . . . . . . . . . . 52

3.5.1 System Model and Simulation Conditions . . . . . . . . . 52

3.5.2 Simulation Results ................ 53

4 Design Procedure for General Cases $\quad 61$

4.1 General Dynamic Model . . . . . . . . . . . . . . . 61

4.2 Adaptive Actuator Failure Compensation . . . . . . . . . . . . . . 64

4.2.1 Nominal Control Design ................ . 64

4.2.2 Adaptive Actuator Failure Compensation Design . . . . . . . 66

4.2.3 Design Procedure for General Case . . . . . . . . . . . . 70

5 Conclusions and Future Work $\quad 73$

5.1 Conclusions . . . . . . . . . . . . . . . . . . . . . . 73

5.2 Future Work . . . . . . . . . . . . . . . . . . 74 


\section{List of Figures}

2.1 second-link planar elbow robotic manipulator. . . . . . . . . . . . 8

3.1 The cooperative robotic system controlling a platform. . . . . . . . . 18

3.2 The height and angle of the platform. . . . . . . . . . . . . . . 19

3.3 System outputs. . . . . . . . . . . . . . . . . . 54

3.4 Tracking error $y_{m}(t)-y(t) \ldots \ldots \ldots \ldots$. . . . . . . . 55

3.5 Adaptive indicator function $\rho_{11}, \rho_{12}, \rho_{13} \ldots \ldots \ldots \ldots$

3.6 Adaptive indicator function $\rho_{21}, \rho_{22}, \rho_{31}, \rho_{32} \ldots \ldots \ldots \ldots$

3.7 Adaptive parameters $\chi_{1(1)(1)}, \chi_{1(1)(2)}, \chi_{1(2)(1)}, \chi_{1(2)(2)} \ldots \ldots \ldots$

3.8 Adaptive parameters $\chi_{2(1)(1)}, \chi_{2(1)(2)}, \chi_{2(2)(1)}, \chi_{2(2)(2)} \ldots \ldots \ldots . . . \quad 59$ 


\section{Chapter 1}

\section{Introduction}

Cooperative manipulator robotic systems play a critical role in many projects such as search and rescue operations, space explorations, and underwater missions. In those applications, robotic systems have to operate in extreme environments $[1,2]$, which actuator failures are likely to occur. Since the robotic systems are used for important tasks [3,4], failures may cause severe consequences. In order for the system to be able to function reliably, we need a controller that can still achieve certain desired properties when failure occurs. There are many ways to design a controller that can improve the robustness of a robotic system $[5,6,7,8,9]$. Although literatures propose different algorithms to solve the problem, most of them rely on knowledge of the actuator failure. However, it may often be difficult to efficiently identify failures within robotic systems in real time. Thus, an adaptive actuator failure compensation scheme, which can operate without the knowledge of actuator failure, is a significant research topic.

\section{$1.1 \quad$ Literature Review}

There are many studies that focus on adaptive actuator failure compensation schemes such as the neural network control [10,11], sliding-mode control [12,13], and an adaptive actuator failure compensation scheme for a near space vehicle [14]. Within 
the robotic field, there are also several important literatures that are directly related to adaptive actuator failure compensation algorithms such as control of a precision pointing hexapod [15], adaptive coordinated controller scheme [16], redundance manipulator system [17]. However, those studies only consider increasing redundancy of the robotic system by using additional manipuilators, which may not be viable in some robotic systems such as in humanoid robots.

In this thesis, we develop a new adaptive actuator failure compensation scheme to control a cooperative manipulator robotic system. The algorithm directly use redundancy of the system from additional joints on a manipulator to ensure desired closed-loop stability and asymptotic output tracking of the system subject to uncertain actuator failure. Since adding joints on a manipulator will significantly change its dynamic model, we will first derive the dynamic model of a cooperative manipulator system with an additional joint. Then, we will design an adaptive actuator failure compensation scheme for the robotic model with redundant actuators.

\subsection{Organization of Thesis}

The thesis is organized as follows. In Chapter II, we introduce the fundamental background in robotic and adaptive control. In Chapter III, we study an adaptive actuator failure compensation scheme based on a cooperative robotic manipulator system. Chapter III is divided into five sections as follows. In Section I, we begin our research by formulating the problem with the discussion of actuator redundancy, control objective, and actuator failure model. In Section II, the dynamic model of a cooperative robotic system is developed using the Euler-Lagrange equation. In Section III, we design a nominal controller for the system in a nominal situation in which the knowledge of actuator failure is known, to construct the controller structure 
and parameterization for failure compensation. In Section IV, the adaptive control scheme is developed based on the nominal controller structure, for the case of uncertain actuator failure. Finally in Section V, the simulation results of the control scheme are presented to confirm the effectiveness of the design. In Chapter IV, we expand our knowledge of the adaptive actuator failure compensation scheme onto a generalize model of cooperative robotic systems by developing a design procedure, which can be used with various cooperative manipulator robotic systems. 


\section{Chapter 2}

\section{Background}

The focus of this thesis is to develop an adaptive actuator failure compensation scheme to control a cooperative manipulators systems subject to uncertain actuator failure, which required background knowledge from robotic and adaptive control field of study. The chapter begin with the discussion of Euler-Lagrange equation, which is a fundametal principle used for developing a dynamic model of a robotic system. We then discuss a simple second-link robotic manipulator, which will provide a general idea about dynamic model of a robotic systems. Finally we will explore the basic technique used in adaptive control and the robotic field.

\subsection{Euler-Lagrange Equation}

Euler-Lagrange equation is one of the most important equation in developing a dynamic model for a robotic system. Because a robotic system is a machanical system, it must follow the principle of conservation of energy. This mean that if there is no external enegy applied to the system, the summation of potential and kinetic energy will always remain the same, regardless of how the system is changed. On the other hand, if we applied any external energy to the system, the change in potential and kinetic energy of the system will be equal to the amount of enegy we put into the system [18]. 
In order to understand how can we use the Euler-Lagrange equation to develop a dynamic model for a robotic system, we start by studying a single-link robotic manipulator system, which is one of the most fundametal system in the robotic field.

Let consider $\theta_{l}, \theta_{m}$ as angles of a link and a motor of a single-link robotic manipulator system. $J_{m}, J_{l}$ as the moment of inertia of the motor and the link accordingly. We clearly can see that the system will has its kinetic energy equation as follows

$$
K=\frac{1}{2} J_{m} \dot{\theta}_{m}^{2}+\frac{1}{2} J_{l} \dot{\theta}_{l}^{2}
$$

Now we consider the potential energy of the system. This potential energy will be depended on the position of the robot. Since we let $\theta_{m}$ be the angle of the system we will need to consider the gear ratio in order to get the real angle of the link $\theta_{l}$. For $n$ represent the gear ratio of the robot arm we can calculate the potential energy as

$$
V=\operatorname{Mgl}\left(1-\cos \frac{\theta_{m}}{n}\right),
$$

where $M$ is the mass of the link, $g$ is the gravity, and $l$ is the length of the robotic arm.

With the potential energy and kinetic energy of the system, we can now derive the total energy of the system. The Lagrangian becomes

$$
L=\frac{1}{2}\left(J_{m}+\frac{J_{l}}{n^{2}}\right) \dot{\theta}_{m}^{2}+\operatorname{Mgl}\left(1-\cos \frac{\theta_{m}}{n}\right) .
$$

In the robotic field we usually write the equation in the following form so that we can analyze the system more effectively, so we will write the dynamic equation in the form

$$
\tau=D(q) \ddot{q}+C(q, \dot{q}) \dot{q}+g(q)
$$


As a result, the Euler-Lagrange equation becomes

$$
\tau=J_{m}+\frac{J_{l}}{n} \ddot{\theta}_{m}+\frac{M g l}{n}\left(\sin \frac{\theta_{m}}{n}\right)
$$

Let $u$ be the input of the system, $B_{m} \dot{\theta}_{m}$ and $B_{l} \dot{\theta}_{l}$ be the damping torques, we will have the dynamic equation for single-link robot manipulatior system as

$$
\begin{aligned}
& \tau=u-\left(B_{m}+\frac{B_{l}}{n^{2}}\right) \dot{\theta_{m}} \\
& u=A \ddot{\theta_{m}}+B \dot{\theta_{m}}+C \sin \left(\frac{\theta_{m}}{n}\right),
\end{aligned}
$$

where

$$
\begin{aligned}
& A=J_{m}+\frac{J_{l}}{n^{2}} \\
& B=B_{m}+\frac{B_{l}}{n^{2}} \\
& C=\frac{M g l}{n} .
\end{aligned}
$$

With the dynamic model for a robotic system, we can start develop a control scheme in order to control the system. 


\subsection{Dynamic Model of A Manipulator System}

In order to gain a better understanding of the dynamic model of robotic system, we expand our knowleage of Euler-Lagrange equation to a more complicated robotic manipulator system, that is the second-link planar elbow robotic manipulator. One thing that we need to keep in mind is that the system of robot manipulator is a non-linear system; however, in this case we can define saparate paremeters to obtain linear relationship between each state of the system [41].

The first step to obtain dynamics of the robot manipulator is to find the EulerLagrange equation for the system. In order to do so, we will need to get kinetic and potential equation of the system similar to the previous case. For planar elbow manipulator with joint angle, we will define the parameters in the system as follows. $\theta_{i}$ will be the angle of each joint, mass and lenght of each link will be represented as $M_{i}$, and $l_{i}$ accordingly. The distance from a joint to the center of mass of that joint will be $l_{c i}$. With this we can derive kinetic enegy from velocity of each link as

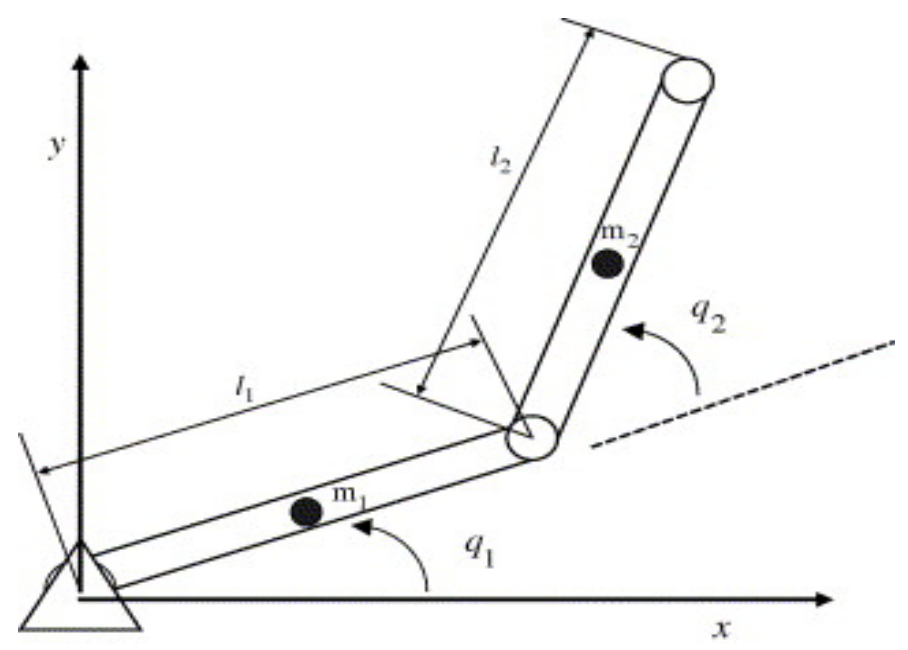

Figure 2.1: second-link planar elbow robotic manipulator. 


$$
v_{c i}=J_{c i} \dot{q},
$$

where $J_{c i}$ represent the jacobian matrix of the system

$$
J_{c 1}=\left[\begin{array}{cc}
-l_{c 1} \sin q_{1} & 0 \\
l_{c 1} \cos q 1 & 0 \\
0 & 0
\end{array}\right] .
$$

For the second link, we have the jacobian matrix as

$$
J_{c 2}=\left[\begin{array}{cc}
-l_{1} \sin q_{1}-l c 2 \sin \left(q_{1}+q_{2}\right) & -l_{c 2} \sin \left(q_{1}+q_{2}\right) \\
l_{1} \cos q_{1}+l c 2 \cos \left(q_{1}+q_{2}\right) & l_{c 2} \cos \left(q_{1}+q_{2}\right) \\
0 & 0
\end{array}\right] .
$$

We can derive matrix $D(q)$ in the robotic dynamics equation as

$$
D(q)=M_{1} J_{c 1}^{T} J_{c 1}+M_{2} J_{c 2}^{T} J_{c 2}+\left[\begin{array}{cc}
I_{1}+I_{2} & I_{2} \\
I_{2} & I_{2}
\end{array}\right]
$$

For potential energy we have the summation of each link in the system

$$
\begin{aligned}
V & =V_{1}+V_{2} \\
& =\left(M_{1} l_{c 1}+M_{2} l_{c 2}\right) g \sin q+M_{2} l_{c 2} g \sin \left(q_{1}+q_{2}\right) .
\end{aligned}
$$


With this matrix $C(q, \dot{q})$ becomes

$$
C=\left[\begin{array}{cc}
\left(-M_{2} l_{1} l_{c 2} \sin q_{2}\right) \dot{q}_{1} & \left(-M_{2} l_{1} l_{c 2} \sin q_{2}\right)\left(q_{1}+q_{2}\right) \\
\left(M_{2} l_{1} l_{c 2} \sin q_{2}\right) \dot{q}_{1} & 0
\end{array}\right] .
$$

The dynamic of the second-link planar system can be written in the form

$$
u=D(q) \ddot{q}+C(q, \dot{q}) \dot{q}+g(q)=Y(q, \dot{q}, \ddot{q}) p .
$$

If we define $p$ as the saparate variables of the system in order to obtain linear relationships of each parameter in the system. We define each value of $p_{i}$ as

$$
\begin{array}{lcc}
p_{1}=M_{1} l_{c 1}^{2} & p_{4}=M_{2} l_{1} L_{c 2} & p_{7}=M_{1} l_{c 1} g \\
p_{2}=M_{2} l_{1}^{2} & p_{5}=I_{1} & p_{8}=M_{2} l_{1} g \\
p_{3}=M_{2} l_{c 2}^{2} & p_{6}=I_{2} & p_{9}=M_{2} l_{c 2} g .
\end{array}
$$

We finally obtain

$$
u=Y(q, \dot{q}, \ddot{q}) p,
$$

where

$$
\begin{gathered}
Y(q, \dot{q}, \ddot{q})=\left[\begin{array}{cccccc}
\ddot{q}_{1} & \ddot{q}_{2} & \ddot{q}_{1}+\ddot{q}_{2} & 2 \cos \left(q_{2} \ddot{q}_{1}\right)+\cos \left(q_{2} \ddot{q}_{2}\right)-2 \sin \left(q_{2} \dot{q}_{1} \dot{q}_{2}\right)-\sin \left(q_{2} \dot{q}_{2}{ }^{2}\right) \\
0 & 0 & \ddot{q}_{1}+\ddot{q}_{2} & & \cos \left(q_{2} \ddot{q}_{1}\right)+\sin q_{2} \\
& & & & \\
& \ddot{q}_{1} & \ddot{q}_{1}+\ddot{q}_{2} & \cos q_{1} & \cos q_{1} & \cos \left(q_{1}+q_{2}\right) \\
& \ddot{q}_{2} & \ddot{q}_{2} & 0 & 0 & \cos \left(q_{1}+q_{2}\right)
\end{array}\right]
\end{gathered}
$$

We can notice that because the system is non-linear, we need to use up to nine 
parameters in order to create a dynamic model that we can work with. In this case we assume that we do not know any information of each parameter. However, usually we can get some information such as the length and mass of each link. With such information we can reduce order of the dynamic system significantly.

\subsection{Simple Joint Control Scheme}

Once of the most important factor in robotic is that we need to be able to control motion of robots, so that it can function properly. In this section, we will investigate a simple control scheme for a robotic manipulator. First we will start by discussing a simple control system. In this case, we will control only one joint in the system with only one motor with a PID controller [41, 49].

A robot system behave similar to any other system that we have learn in control theory, in which the plant of the system will be a robotic system. As a result, we will first derive the dynamic equation of the system. When $J_{m}$ represent the sum of the actuator and gear inertia, and $B_{m}$ is the motor friction. A DC motor system will have its dynamic equation in laplace domain as

$$
\frac{1}{J_{m} s^{2}+B_{m} s}
$$

We can apply a PID compensator to the system as

$$
C(s)=K_{p}+\frac{K_{I}}{s}+K_{D} s
$$

As a result, we get the close loop equation of the system as follow

$$
Y(s)=\frac{K_{D} s^{2}+K_{p} s+K_{I}}{J_{m} s^{3}+\left(B_{m}+K_{D}\right) s^{2}+K_{p} s+K_{I}} R(s) .
$$


We know that in robotic the accuracy and repeatability of the system are the most important properties of the system. The system must be able to accurately track the reference signal or reach the desire position as intended. As we can see in the previous example, a PID controller is good enough to achieve those properties for a single motor in the system. The closed loop system equation show that the PID controller will provide the output tracking and it can also reject some disturbance in the system. However, in a more complicated system with many links, we will need to use more advance technique to ensure the accuracy and repeatability of the robotic system.

\subsection{Adaptive Control for Robotic System}

In this section we discuss a basic adaptive control scheme in robotic. In a robotic system a robot need to be able to function in real environment subject to some uncertainties in the system. This mean that many times we do not know information

of one or more parameters in the system; as a result, we have to develop an adaptive controller in order to compensate for those unknown parameters [41].

The basic idea of adaptive control is to adjust control parameters in a system according to the feedback signal. Most of the time, we will try to adjust the system parameters in a way such that the output of the system becomes closer to the reference signal. With adaptive control we can ensure the system stability, as well as improve robustness of robotic systems.

In this chapter, we will use second-link robotic manipulater that we derive ealier as an example in our discussion. From section 2.2 we have the dynamic model of 
second-link robot manipulator as

$$
\begin{aligned}
& u=D(q) \ddot{q}+C(q, \dot{q}) \dot{q}+g(q)=Y(q, \dot{q}, \ddot{q}) p \\
& Y(q, \dot{q}, \ddot{q})=\left[\begin{array}{cccc}
\ddot{q}_{1} & \ddot{q}_{2} & \ddot{q}_{1}+\ddot{q}_{2} & 2 \cos \left(q_{2} \ddot{q}_{1}\right)+\cos \left(q_{2} \ddot{q}_{2}\right)-2 \sin \left(q_{2} \dot{q}_{1} \dot{q}_{2}\right)-\sin \left(q_{2} \dot{q}_{2}{ }^{2}\right) \\
0 & 0 & \ddot{q}_{1}+\ddot{q}_{2} & \cos \left(q_{2} \ddot{q}_{1}\right)+\sin q_{2}
\end{array}\right. \\
& \left.\begin{array}{ccccc}
\ddot{q}_{1} & \ddot{q}_{1}+\ddot{q}_{2} & \cos q_{1} & \cos q_{1} & \cos \left(q_{1}+q_{2}\right) \\
\ddot{q}_{2} & \ddot{q}_{2} & 0 & 0 & \cos \left(q_{1}+q_{2}\right)
\end{array}\right] .
\end{aligned}
$$

One possibility of an adaptive control scheme that can guarantee the system stability and tracking could be designed as follows. First we define a controller structure based on the dynamic equation of the system. We will use $\theta(t)$ as the system parameter estimators and a constant matrix $K_{D}$ as a design matrix. With $s(t)=\dot{e}+\Lambda_{0} e$, $e=q-q_{d}$, where $\Lambda_{0}$ be a constant matrix whose eigenvalue have positive real part, we have the control law

$$
u=Y(q, \dot{q}, \ddot{q}) \theta(t)-K_{D} s(t), 0<K_{D}=K_{D}^{T} \in R^{n x n}
$$

Since our adaptive controller will need to adapt in order to make the system track the reference signal, we define an adaptive law that we will use to update $\theta(t)$ as

$$
\dot{\theta}(t)=-\Gamma^{-1} Y^{T}(q, \dot{q}, \ddot{q}) s(t), 0<\Gamma=\Gamma^{T} \in R^{n_{\theta} x n_{\theta}}
$$

With this controller and the adaptive law, its can be proven that the control scheme can guarantee the stability and asymptotic tracking of the system. 


\subsection{Backstepping Nonlinear Control Design}

In order to control a nonlinear system, we need to construct a nonlinear controller for the system. In this section, we consider using a backstepping design to develop a controller for a second-order nonlinear system [18, 44].

Consider a second order nonlinear system

$$
\begin{aligned}
& \dot{x}_{1}=f\left(x_{1}\right) x_{2} \\
& \dot{x}_{2}=g\left(x_{1}, x_{2}\right)+u(t) \\
& y=x_{1}
\end{aligned}
$$

where $x_{1}, x_{2}$ is the state, $y$ is the output, and $u$ is the input of the system.

The objective is to design a state feedback control $u(t)$ such that all closed-loop signal are bounded and the system output track a given reference output signal $y_{m}(t)$. For a second order system, the backstepping design can be done with two design steps.

Step 1: Define $z_{1}=x_{1}-y_{m}, z_{2}=x_{2}-\beta$, where $\beta$ is a design function to be determined. From the state equation of the system, we can write $\dot{z}_{1}$ as

$$
\begin{aligned}
\dot{z}_{1} & =\dot{x}_{1}-\dot{y}_{m} \\
& =f\left(x_{1}\right) x_{2}-\dot{y}_{m} \\
& =f\left(x_{1}\right)\left(z_{2}-\beta\right)-\dot{y}_{m} .
\end{aligned}
$$

In order to choose $\beta$ to stablize the system, we consider a Lyapunov candidate function 
and its derivative

$$
\begin{aligned}
& V_{1}=\frac{1}{2} z_{1}^{T} z_{1} \\
& \dot{V}_{1}=z_{1}\left(f\left(x_{1}\right) z_{2}+f\left(x_{1}\right) \beta-\dot{y}_{m}\right) .
\end{aligned}
$$

With the design function

$$
\beta=f\left(x_{1}\right)^{-1}\left(-c_{1} z_{1}+\dot{y}_{m}\right),
$$

where $c_{1}$ is a positive constant. The derivative of the Lyapunov function becomes

$$
\dot{V}_{1}=-c_{1} z_{1}^{T} z_{1}+f\left(x_{1}\right) z_{1} z_{2} .
$$

We see that the term $-c_{1} z_{1}^{T} z_{1}$ is always negative.

Step 2: Since $z_{2} \neq 0$, we continue using backstepping design by considering

$$
\begin{aligned}
\dot{z}_{2} & =\dot{x}_{2}-\dot{\beta} \\
& =g\left(x_{1}, x_{2}\right)+u(t)-\dot{\beta},
\end{aligned}
$$

and the second Lyapunov function

$$
V_{2}=V_{1}+\frac{1}{2} z_{2}^{T} z_{2} .
$$

The time-derivative of $V_{2}$ is

$$
\begin{aligned}
\dot{V}_{2} & =\dot{V}_{1}+z_{2}^{T} \dot{z}_{2} \\
& =-c_{1} z_{1}^{T} z_{1}+z_{2}^{T}\left(f\left(x_{1}\right) z_{1}+g\left(x_{1}, x_{2}\right)+u(t)-\dot{\beta}\right)
\end{aligned}
$$


As a result, the control signal $u(t)$ is chosen as

$$
u(t)=-g\left(x_{1}, x_{2}\right)-c_{2} z_{2}-f\left(x_{1}\right) z_{1}+\dot{\beta} .
$$

With the chosen $u(t)$, equation (2.41) becomes

$$
\dot{V}_{2}=-c_{1} z_{1}^{T} z_{1}-c_{2} z_{2}^{T} z_{2}
$$

With a positive constant $c_{2}$, we have $\dot{V}_{2}$ is negative semidefinite and only equal to zero when $z_{1}=z_{2}=0$. With this design, the nonlinear can guarantee the closed-loop signal boundedness and asymptotic output tracking of the system: $\lim _{t \rightarrow \infty}(y(t)-$ $\left.y_{m}(t)\right)=0$. 


\section{Chapter 3}

\section{Adaptive Actuator Failure Compen- sation for A Cooperative Manipula- tor System}

The objective of this study is to design an adaptive actuator failure compensation scheme for a robotic system without the knowledge of failing actuators. In this study we use a two-dimensional cooperative manipulator system. The system contains two manipulators, which are attached to each side of a rigid platform as shown in Figure 3.1. We design a controller that can guarantee asymptotic tracking of both height $h(t)$ and angle $\theta(t)$ of the system. The robotic system can be extended to various robotic applications that use a cooperative manipulator system such as a hexapod system or the lower part of a humanoid robot.

\subsection{Problem Formulation}

In this section, we discuss several important topics that are needed in order to solve the problem, which are actuator redundancy in the system, the control objective, and the actuator failure model used in the study. 


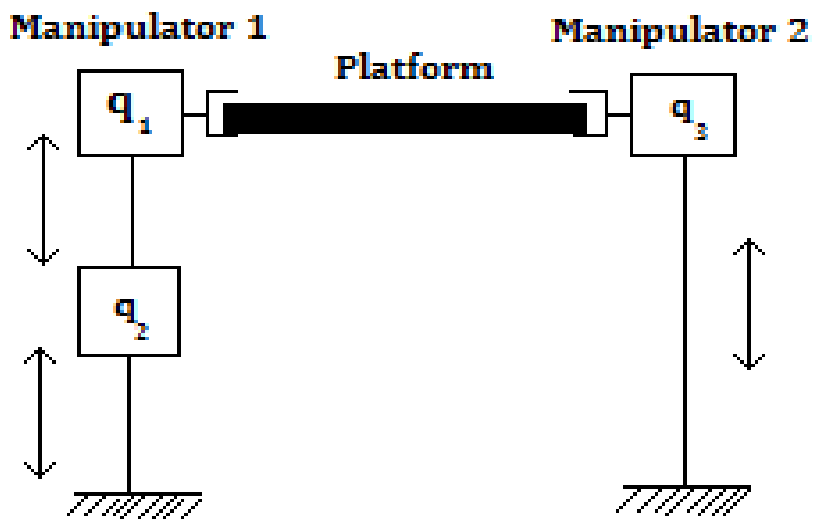

Figure 3.1: The cooperative robotic system controlling a platform.

\subsubsection{Actuator Redundancy}

A system with two degrees of freedom needs two actuators in order to control the system; however, for a system to be able to operate when an actuator failure occurs, redundancy in the system is needed.

In many systems such as a humanoid robot, adding another leg to the robot for redundancy is not possible without the loss of functionality of the system. In our robotic system model, the redundancy of the system come from an additional joint in the left manipulator. As in Figure 3.1, the system uses three actuators $q_{1}, q_{2}, q_{3}$ to support a rigid platform that links actuator $q_{1}$ and $q_{3}$ together. The actuator $q_{2}$ is added to increase redundancy in the system, so that we can develop an actuator failure compensation scheme to compensate for possible actuator failure that could occur on the left side of the platform.

\subsubsection{Control Objective}

The objective of the control scheme is to guarantee positional tracking of the height $h(t)$, and angle $\theta(t)$ of the system subject to uncertain actuator failure. 


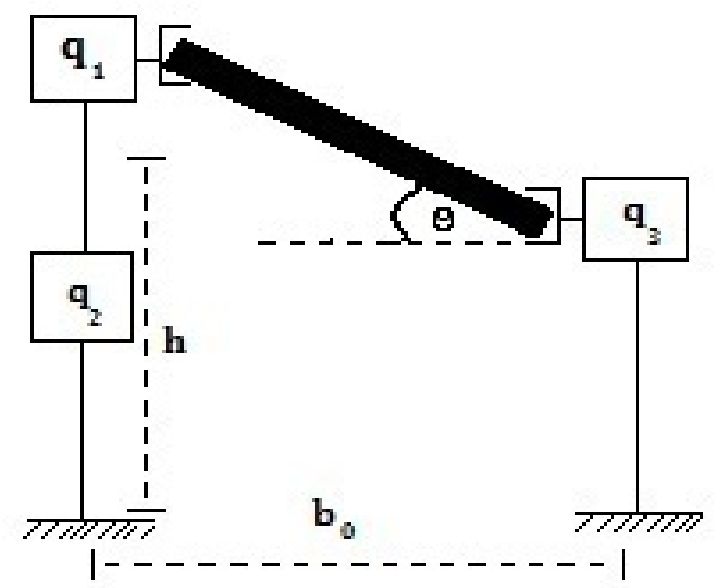

Figure 3.2: The height and angle of the platform.

The development of the adaptive control algorithm can be divided into three parts. First we will find a feedback control signal $\omega_{d}$ from a backstepping control design, which ensures the stability of the system in the absence of actuator failure. Then, the nominal controller for the system will be developed based on the knowledge of actuator failure. The nominal control will be designed such that the control signal matches the signal $\omega_{d}$, so that the output of the system follows the desirable trajectory. Finally, the adaptive actuator failure compensation scheme is developed based on the nominal controller structure, using parameter adaptation to handle the actuator failure uncertainties.

\subsubsection{Actuator Failure Model}

When an actuator failure occurs in the system, the control input $u_{j}(t)$ associated with the failing actuator $j$ may become an arbitrary value, which can be denoted as

$$
u_{j}(t)=\bar{u}_{j}(t), t \geq t_{j}, j \in\{1,2, \ldots, m\} .
$$


Although we do not know the value of the control input when failure occurs, we can model the failure based on some knowledge of the structure of the actuator as

$$
\bar{u}_{j}(t)=\bar{u}_{j 0}+\sum_{i=1}^{n_{j}} \bar{u}_{i j} f_{a i j}(t), t \geq t_{j},
$$

where $n_{j}$ is the number of components in the actuator, $f_{a i j}$ is a known function corresponding to each component of the actuator, and $j, t_{j}, \bar{u}_{i j}$ are unknown actuator, time, and failure of each component of the actuator. In this study, we consider three cases of possible actuator failure patterns: no failure occurs, actuator $u_{1}$ fails, or actuator $u_{2}$ fails. 


\subsection{Derivation of the Dynamic Model}

The dynamic model of a redundant manipulator robotic system has been discussed in various books and published papers $[18,19]$. There are several approaches that can be used to obtain a set of dynamic equations of the system such as using Newton's second law of motion, or the Euler-Lagrange equation. Newton's second law of motion is a well-known method of analyzing movement of an object. However, in order to calculate the dynamic of an object, one needs to know every force that acts on the object. In robotic systems, which contain many joints and links, it is complicated to calculate constraint forces in the system. As a result, using the Euler-Lagrange equation to develop the dynamic equations is a preferable method.

In this study, the derivation of the dynamic model of the system is divided in three parts. First we derive dynamic equations of the system using the Euler-Lagrange equation. After we obtain the dynamic equations, state parameters of the system are defined and calculated. Finally, the dynamic equation is put into the state space form, so that it can be used for the development of the adaptive actuator failure compensation scheme.

\subsubsection{Euler-Lagrange Equation}

In order to use the Euler-Lagrange equation, we need to determine the generalized coordinates for the system [18]. There are two sets of independent coordinates that should be considered. The first set of generalized coordinates is to use height the $h(t)$ and angle $\theta(t)$ of the platform as the generalized coordinates of the system. Although outputs of the system will be directly related to each coordinate, the dynamic equations are more complicated because the force of each actuator will act on different directions to each coordinate. On the other hand, by selecting the position of each 
actuator $q_{1}, q_{2}, q_{3}$ as the generalized coordinates, we can reduce the complexity of the derivation; as a result, we will use this second set as the generalized coordinates.

Before we derive the dynamic equations of the system, we consider the relationship between the coordinates and the outputs of the system.

From Figure 2, we can write the height $h$ and the angle $\theta$ of the platform in term of the position of each actuator as

$$
\begin{aligned}
& h\left(q_{1}, q_{2}, q_{3}\right)=\frac{q_{1}+q_{2}+q_{3}}{2} \\
& \theta\left(q_{1}, q_{2}, q_{3}\right)=\arctan \left(\frac{q_{1}+q_{2}-q_{3}}{b_{0}}\right),
\end{aligned}
$$

where the constant $b_{0}$ is the lenght of the base of the platform. The derivatives of (3.3) and (3.4) are

$$
\begin{aligned}
\dot{h}\left(q_{1}, q_{2}, q_{3}\right) & =\frac{1}{2}\left(\dot{q}_{1}+\dot{q}_{2}+\dot{q}_{3}\right) \\
\dot{\theta}\left(q_{1}, q_{2}, q_{3}\right) & =\frac{b_{0}\left(\dot{q}_{1}+\dot{q}_{2}-\dot{q}_{3}\right)}{b_{0}^{2}+\left(q_{1}+q_{2}-q_{3}\right)^{2}} .
\end{aligned}
$$

Based on the generalize coordinate $q_{i}$ for $i=1,2,3$, we consider the Lagrange's equation

$$
\frac{d}{d t}\left(\frac{\partial L}{\partial \dot{q}_{i}}\right)-\frac{\partial L}{\partial q_{i}}=\tau_{i}
$$

where the Lagrangian $L=T-V$, $\mathrm{T}$ is the kinetic energy, $\mathrm{V}$ is the potential energy, $q_{i}=\left[q_{1}, q_{2}, q_{3}\right]$ are the vectors of generalized coordinates, and $u_{i}$ is the torque of each actuator, which acts along each coordinate. If the dependency forces between $q_{1}, q_{2}$ are small, we can approximate that the generalized coordinates are independant from 
each other. Thus, the kinetic energy and the potential energy can be written as

$$
\begin{gathered}
T=\frac{1}{2}\left(m_{1} \dot{q}_{1}^{2}+\left(m_{1}+m_{2}\right) \dot{q}_{2}^{2}+m_{3} \dot{q}_{3}^{2}\right) \\
+\frac{1}{2} m_{p} \dot{h}^{2}+\frac{1}{2} I_{p} \dot{\theta}^{2} \\
V=m_{1} g q_{1}+\left(m_{1}+m_{2}\right) g q_{2}+m_{3} g q_{3}+m_{p} g h,
\end{gathered}
$$

where $\mathrm{g}$ is the scalar value of the gravity, $m_{i}$ is the mass of each actuator, $m_{p}$ is the mass of the platform, and $I_{p}$ is the moment of inertia of the platform. We calculate each term in the Lagrange's equation as

$$
\frac{\partial L}{\partial q_{i}}=-\left(m_{i} g+\frac{1}{2} m_{p} g\right) ; i=1,2,3
$$

For the term $\frac{d}{d t} \frac{\partial L}{\partial \dot{q}_{i}}$, we first derive $\frac{\partial L}{\partial \dot{q}_{i}}$ as

$$
\begin{aligned}
& \frac{\partial L}{\partial \dot{q}_{1}}=m_{1} \dot{q}_{1}+m_{p} \dot{h} \frac{d \dot{h}}{d \dot{q}_{1}}+I_{p} \dot{\theta} \frac{d \dot{\theta}}{d \dot{q}_{1}} \\
& \frac{\partial L}{\partial \dot{q}_{2}}=\left(m_{1}+m_{2}\right) \dot{q}_{2}+m_{p} \dot{h} \frac{d \dot{h}}{d \dot{q}_{2}}+I_{p} \dot{\theta} \frac{d \dot{\theta}}{d \dot{q}_{2}} \\
& \frac{\partial L}{\partial \dot{q}_{3}}=m_{3} \dot{q}_{3}+m_{p} \dot{h} \frac{d \dot{h}}{d \dot{q}_{3}}+I_{p} \dot{\theta} \frac{d \dot{\theta}}{d \dot{q}_{3}} .
\end{aligned}
$$

From equation (3.5) we have $\frac{d \dot{h}}{d \dot{q}_{i}}=\frac{1}{2}$, and

$$
m_{p} \dot{h} \frac{d \dot{h}}{d \dot{q}_{i}}=\frac{1}{4} m_{p}\left(\dot{q}_{1}+\dot{q}_{2}+\dot{q}_{3}\right) .
$$


Consider $\dot{\theta} \frac{d \dot{\theta}}{d \dot{q}_{i}}$ based on equation (3.6), we have

$$
\dot{\theta} \frac{d \dot{\theta}}{d \dot{q}_{i}}=\frac{\alpha_{i} b_{0}^{2}\left(\dot{q}_{1}+\dot{q}_{2}-\dot{q}_{3}\right)}{\left(b_{0}^{2}+\left(q_{1}+q_{2}-q_{3}\right)^{2}\right)^{2}}
$$

where the constant $\alpha_{i}=1$ for $i=1,2, \alpha_{i}=-1$ for $i=3$. Define $\bar{q}_{1}=q_{1}+q_{2}+q_{3}$, $\bar{q}_{2}=q_{1}+q_{2}-q_{3}$, and $\Psi_{1}, \Psi_{2, i}$ as

$$
\begin{aligned}
\Psi_{1} & =\frac{1}{4} m_{p}\left(\dot{\bar{q}}_{1}\right) \\
\Psi_{2, i} & =\frac{\alpha_{i} b_{0}^{2} I_{p} \dot{\bar{q}}_{2}}{\left(b_{0}^{2}+\bar{q}_{2}^{2}\right)^{2}} .
\end{aligned}
$$

Then, the time derivative $\frac{d}{d t}\left(\frac{\partial L}{\partial \dot{q}_{i}}\right)$ can be written as

$$
\begin{aligned}
& \frac{d}{d t} \frac{\partial L}{\partial \dot{q}_{1}}=m_{1} \ddot{q}_{1}+\dot{\Psi}_{1}+\dot{\Psi}_{2,1} \\
& \frac{d}{d t} \frac{\partial L}{\partial \dot{q}_{2}}=\left(m_{1}+m_{2}\right) \ddot{q}_{2}+\dot{\Psi}_{1}+\dot{\Psi}_{2,2} \\
& \frac{d}{d t} \frac{\partial L}{\partial \dot{q}_{3}}=m_{3} \ddot{q}_{3}+\dot{\Psi}_{1}+\dot{\Psi}_{2,3},
\end{aligned}
$$

where $\dot{\Psi}_{1}, \dot{\Psi}_{1}$ are expressed as

$$
\begin{aligned}
\dot{\Psi}_{1} & =\frac{1}{4} m_{p}\left(\ddot{\bar{q}}_{1}\right) \\
\dot{\Psi}_{2, i} & =\alpha_{i} b_{0}^{2} I_{p}\left(\frac{\ddot{\bar{q}}_{2}}{\left(b_{0}^{2}+\bar{q}_{2}^{2}\right)^{2}}-\frac{4 \bar{q}_{2} \dot{\bar{q}}_{2}^{2}}{\left(b_{0}^{2}+\bar{q}_{2}^{2}\right)^{3}}\right)
\end{aligned}
$$

Finally, the Lagrange's equation becomes

$$
\begin{aligned}
m_{1} \ddot{q}_{1}+\dot{\Psi}_{1}+\dot{\Psi}_{2,1}+\left(m_{1} g+\frac{1}{2} m_{p} g\right) & =\tau_{1} \\
\left(m_{1}+m_{2}\right)\left(\ddot{q}_{2}+g\right)+\dot{\Psi}_{1}+\dot{\Psi}_{2,2}+\frac{1}{2} m_{p} g & =\tau_{2} \\
m_{3} \ddot{q}_{3}+\dot{\Psi}_{1}+\dot{\Psi}_{2,3}+\left(m_{3} g+\frac{1}{2} m_{p} g\right) & =\tau_{3} .
\end{aligned}
$$




\subsubsection{Determination of Signals $\ddot{\bar{q}}_{1}$ and $\ddot{\bar{q}}_{2}$}

In order to put the dynamic equations in the state space form, we need to rewrite the dynamic equations in term of $\ddot{\bar{q}}_{1}$ and $\ddot{\bar{q}}_{2}$. Rewrite equations (3.23)- (3.25) for each $q_{i}$ as

$$
\begin{aligned}
& \ddot{q}_{1}=\frac{\tau_{1}-\left(\dot{\Psi}_{1}+\dot{\Psi}_{2,1}+\left(m_{1} g+\frac{1}{2} m_{p} g\right)\right)}{m_{1}} \\
& \ddot{q}_{2}=\frac{\tau_{2}-\dot{\Psi}_{1}-\dot{\Psi}_{2,2}}{m_{1}+m_{2}}-\frac{\left(\left(m_{1}+m_{2}\right) g+\frac{1}{2} m_{p} g\right)}{m_{1}+m_{2}} \\
& \ddot{q}_{3}=\frac{\tau_{3}-\left(\dot{\Psi}_{1}+\dot{\Psi}_{2,3}+\left(m_{3} g+\frac{1}{2} m_{p} g\right)\right)}{m_{3}} .
\end{aligned}
$$

Define a parameter $\zeta_{1}=1+\frac{m_{p}}{4}\left(\frac{1}{m_{1}}+\frac{1}{m_{1}+m_{2}}+\frac{1}{m_{3}}\right)$. We can combine equation (3.26)- (3.28)) to get $\ddot{\bar{q}}_{1}$ as

$$
\begin{aligned}
\ddot{\bar{q}}_{1}= & \frac{1}{\zeta_{1}}\left(\left(\frac{\tau_{1}}{m_{1}}+\frac{\tau_{2}}{m_{1}+m_{2}}+\frac{\tau_{3}}{m_{3}}\right)\right. \\
& -\left(3 g+\frac{m_{p} g}{2}\right)\left(\frac{1}{m_{1}}+\frac{1}{m_{1}+m_{2}}+\frac{1}{m_{3}}\right) \\
& \left.-\dot{\Psi}_{2,1}\left(\frac{1}{m_{1}}+\frac{1}{m_{1}+m_{2}}+\frac{1}{m_{3}}\right)\right) .
\end{aligned}
$$

With a parameter $\zeta_{2}=\left(1+\frac{b_{0}^{2} I_{p}}{\left(b_{0}^{2}+\bar{q}_{2}^{2}\right)^{2}}\left(\frac{1}{m_{1}}+\frac{1}{m_{1}+m_{2}}+\frac{1}{m_{3}}\right)\right)$, we also have $\ddot{\bar{q}}_{2}$ as

$$
\begin{aligned}
\ddot{\bar{q}}_{2}= & \frac{1}{\zeta_{2}}\left(\left(\frac{\tau_{1}}{m_{1}}+\frac{\tau_{2}}{m_{1}+m_{2}}-\frac{\tau_{3}}{m_{3}}\right)-\dot{\Psi}_{1}\left(\frac{1}{m_{1}}+\frac{1}{m_{1}+m_{2}}-\frac{1}{m_{3}}\right)\right. \\
& -\left(g+\frac{m_{p} g}{2}\right)\left(\frac{1}{m_{1}}+\frac{1}{m_{1}+m_{2}}-\frac{1}{m_{3}}\right) \\
& \left.-\frac{4 b_{0}^{2} I_{p}\left(\bar{q}_{2} \dot{\bar{q}}_{2}^{2}\right)}{b_{0}^{2}+\bar{q}_{2}^{2}}\left(\frac{1}{m_{1}}+\frac{1}{m_{1}+m_{2}}+\frac{1}{m_{3}}\right)\right) .
\end{aligned}
$$

Let parameters $M_{a}=\left(\frac{1}{m_{1}}+\frac{1}{m_{1}+m_{2}}+\frac{1}{m_{3}}\right)$, and $M_{b}=\left(\frac{1}{m_{1}}+\frac{1}{m_{1}+m_{2}}-\frac{1}{m_{3}}\right)$. Since 
$\Psi_{1}, \Psi_{2,1}$ contain $\ddot{\bar{q}}_{1}, \ddot{\bar{q}}_{2}$, we rewrite equation (3.29), and (3.30) as

$$
\begin{gathered}
\ddot{\bar{q}}_{1}=\frac{1}{\zeta_{1}}\left(\left(\frac{\tau_{1}}{m_{1}}+\frac{\tau_{2}}{m_{1}+m_{2}}+\frac{\tau_{3}}{m_{3}}\right)\right. \\
\left.-\left(3 g+\frac{m_{p} g}{2}+\frac{b_{0}^{2} I_{p} \ddot{\bar{q}}_{2}}{\left(b_{0}^{2}+\bar{q}_{2}^{2}\right)^{2}}-\frac{4 b_{0}^{2} I_{p} \bar{q}_{2} \dot{\bar{q}}_{2}^{2}}{\left(b_{0}^{2}+\bar{q}_{2}^{2}\right)^{3}}\right) M_{a}\right) \\
\ddot{\bar{q}}_{2}=\frac{1}{\zeta_{2}}\left(\left(\frac{\tau_{1}}{m_{1}}+\frac{\tau_{2}}{m_{1}+m_{2}}-\frac{\tau_{3}}{m_{3}}-\frac{1}{4} m_{p}\left(\ddot{\bar{q}}_{1}\right)\right)\right. \\
\left.-\left(\left(g+\frac{m_{p} g}{2}\right)\right) M_{b}+\frac{4 b_{0}^{2} I_{p}\left(\bar{q}_{2} \dot{\bar{q}}_{2}^{2}\right)}{b_{0}^{2}+\bar{q}_{2}^{2}} M_{a}\right) .
\end{gathered}
$$

With $\zeta_{3}=\frac{b_{0}^{2} I_{p}}{\left(b_{0}^{2}+\bar{q}_{2}^{2}\right)^{2}} M_{a}$, by substitution of $\ddot{\bar{q}}_{2}$ from (3.31), equation (3.32) becomes

$$
\begin{aligned}
\ddot{\bar{q}}_{1}= & \frac{1}{\zeta_{1}}\left[\left(\frac{\tau_{1}}{m_{1}}+\frac{\tau_{2}}{m_{1}+m_{2}}+\frac{\tau_{3}}{m_{3}}\right)\right. \\
& -\frac{\zeta_{3}}{\zeta_{2}}\left(\frac{\tau_{1}}{m_{1}}+\frac{\tau_{2}}{m_{1}+m_{2}}-\frac{\tau_{3}}{m_{3}}\right) \\
& +\frac{\zeta_{3}}{\zeta_{2}}\left(\frac{1}{4} m_{p}\left(\ddot{\bar{q}}_{1}\right)+\left(g+\frac{m_{p} g}{2}\right)\right) M_{b}-\left(3 g+\frac{m_{p} g}{2}\right) \\
& \left.-\left(\frac{\zeta_{3}}{\zeta_{2}} \frac{4 b_{0}^{2} I_{p}\left(\bar{q}_{2} \dot{q}_{2}^{2}\right)}{b_{0}^{2}+\bar{q}_{2}^{2}}-\frac{4 b_{0}^{2} I_{p} \bar{q}_{2} \dot{\bar{q}}_{2}^{2}}{\left(b_{0}^{2}+\bar{q}_{2}^{2}\right)^{3}}\right) M_{a}\right]
\end{aligned}
$$

With $\zeta_{4}=1+\frac{m_{p} \zeta_{3}}{4 \zeta_{1} \zeta_{2}} M_{b}$, we have

$$
\begin{aligned}
\ddot{\bar{q}}_{1}= & \frac{1}{\zeta_{4} \zeta_{1}}\left[\left(\frac{\tau_{1}}{m_{1}}+\frac{\tau_{2}}{m_{1}+m_{2}}+\frac{\tau_{3}}{m_{3}}\right)\right. \\
& -\frac{\zeta_{3}}{\zeta_{2}}\left(\frac{\tau_{1}}{m_{1}}+\frac{\tau_{2}}{m_{1}+m_{2}}-\frac{\tau_{3}}{m_{3}}\right) \\
& +\frac{\zeta_{3}}{\zeta_{2}}\left(g+\frac{m_{p} g}{2}\right) M_{b}-\left(3 g+\frac{m_{p} g}{2}\right) M_{a} \\
& \left.-\left(\frac{\zeta_{3}}{\zeta_{2}} \frac{4 b_{0}^{2} I_{p}\left(\bar{q}_{2} \dot{\bar{q}}_{2}^{2}\right)}{b_{0}^{2}+\bar{q}_{2}^{2}}-\frac{4 b_{0}^{2} I_{p} \bar{q}_{2} \dot{\bar{q}}_{2}^{2}}{\left(b_{0}^{2}+\bar{q}_{2}^{2}\right)^{3}}\right) M_{a}\right] .
\end{aligned}
$$

We solve for $\ddot{\bar{q}}_{2}$ with a similar approach. With $\zeta_{5}=\frac{m_{p}}{4} M_{b}$, by substitution of $\ddot{\bar{q}}_{1}$ from 
equation (3.33), equation (3.34) becomes

$$
\begin{aligned}
\ddot{\bar{q}}_{2}= & \frac{1}{\zeta_{2}}\left(\frac{\tau_{1}}{m_{1}}+\frac{\tau_{2}}{m_{1}+m_{2}}-\frac{\tau_{3}}{m_{3}}\right) \\
& -\frac{1}{\zeta_{2}}\left(\left(g+\frac{m_{p} g}{2}\right) M_{b}+\frac{4 b_{0}^{2} I_{p}\left(\bar{q}_{2} \dot{\bar{q}}_{2}^{2}\right)}{b_{0}^{2}+\bar{q}_{2}^{2}} M_{a}\right) \\
& -\frac{\zeta_{5}}{\zeta_{4} \zeta_{1} \zeta_{2}}\left[\left(\frac{\tau_{1}}{m_{1}}+\frac{\tau_{2}}{m_{1}+m_{2}}+\frac{\tau_{3}}{m_{3}}\right)\right. \\
& +\frac{\zeta_{3}}{\zeta_{2}}\left(\frac{\tau_{1}}{m_{1}}+\frac{\tau_{2}}{m_{1}+m_{2}}-\frac{\tau_{3}}{m_{3}}\right) \\
& +\frac{\zeta_{3}}{\zeta_{2}}\left(g+\frac{m_{p} g}{2}\right) M_{b}+\left(3 g+\frac{m_{p} g}{2}\right) M_{a} \\
& \left.+\frac{\zeta_{3}}{\zeta_{2}}\left(\frac{4 b_{0}^{2} I_{p}\left(\bar{q}_{2} \dot{\bar{q}}_{2}^{2}\right)}{b_{0}^{2}+\bar{q}^{2}}-\frac{4 b_{0}^{2} I_{p} \bar{q}_{2} \dot{\bar{q}}_{2}^{2}}{\left(b_{0}^{2}+\bar{q}_{2}^{2}\right)^{3}}\right) M_{a}\right] .
\end{aligned}
$$

\subsubsection{Dynamic Model in State Space Form}

The dynamic equations can now be written in the state space form. We define $x_{1}=$ $\left[x_{11}, x_{12}\right]^{T}=[h, \theta]^{T}$, and $x_{2}=\left[x_{21}, x_{22}\right]^{T}=\left[\dot{\bar{q}}_{1}, \dot{\bar{q}}_{2}\right] \cdot{ }^{T}$ With $\bar{q}_{1}=2 h, \bar{q}_{2}=b_{0} \tan \theta$, we have

$$
\begin{gathered}
\dot{x}_{1}=\left[\begin{array}{cc}
\frac{1}{2} \dot{\bar{q}}_{1} & \frac{1}{b_{0}\left(1+\theta^{2}\right)} \dot{\bar{q}}_{2}
\end{array}\right]^{T} \\
\dot{x}_{2}=\left[\begin{array}{ll}
\left(\ddot{q}_{1}+\ddot{q}_{2}+\ddot{q}_{3}\right) & \left(\ddot{q}_{1}+\ddot{q}_{2}-\ddot{q}_{3}\right)
\end{array}\right]^{T} .
\end{gathered}
$$

Let $u=\left[\tau_{1}, \tau_{2}, \tau_{3}\right]^{T}$ be the system input, and $y=[h, \theta]^{T}$ be the system output. The state equation can be written as

$$
\begin{aligned}
\dot{x}_{1} & =f\left(x_{1}\right) x_{2} \\
\dot{x}_{2} & =g_{1}\left(x_{1}, x_{2}\right)+g_{2}\left(x_{1}, x_{2}\right) u \\
y & =x_{1},
\end{aligned}
$$


where the matrix $f\left(x_{1}\right)$ is expressed as

$$
f\left(x_{1}\right)=\left[\begin{array}{cc}
f_{11}\left(x_{1}\right) & 0 \\
0 & f_{22}\left(x_{1}\right)
\end{array}\right] .
$$

Based on equation (3.36), we obtain

$$
f_{11}\left(x_{1}\right)=\frac{1}{2}, \quad f_{22}\left(x_{1}\right)=\frac{1}{b_{0}\left(1+x_{12}^{2}\right)}
$$

Let $g_{1}\left(x_{1}, x_{2}\right)=\left[g_{11}\left(x_{1}, x_{2}\right), g_{12}\left(x_{1}, x_{2}\right)\right] .{ }^{T}$ From equations (3.34) and (3.35), we have

$$
\begin{aligned}
g_{11}= & \frac{1}{\zeta_{4} \zeta_{1}}\left[\frac{\zeta_{3}}{\zeta_{2}}\left(g+\frac{m_{p} g}{2}\right) M_{b}-\left(\left(3 g+\frac{m_{p} g}{2}\right)\right.\right. \\
& \left.\left.+\frac{\zeta_{3}}{\zeta_{2}} \frac{4 b_{0}^{2} I_{p}\left(\bar{q}_{2} x_{22}^{2}\right)}{b_{0}^{2}+\bar{q}_{2}^{2}}-\frac{4 b_{0}^{2} I_{p} \bar{q}_{2} x_{22}^{2}}{\left(b_{0}^{2}+\bar{q}_{2}^{2}\right)^{3}}\right) M_{a}\right] \\
g_{12}= & \frac{1}{\zeta_{2}}\left[-\left(g+\frac{m_{p} g}{2}\right) M_{b}-\frac{4 b_{0}^{2} I_{p}\left(\bar{q}_{2} x_{22}^{2}\right)}{b_{0}^{2}+\bar{q}_{2}^{2}} M_{a}\right] \\
& -\frac{\zeta_{5}}{\zeta_{4} \zeta_{1} \zeta_{2}}\left[\frac{\zeta_{3}}{\zeta_{2}}\left(g+\frac{m_{p} g}{2}\right) M_{b}+\left(\left(3 g+\frac{m_{p} g}{2}\right)\right.\right. \\
& \left.\left.+\frac{\zeta_{3}}{\zeta_{2}} \frac{4 b_{0}^{2} I_{p}\left(\bar{q}_{2} x_{22}^{2}\right)}{b_{0}^{2}+\bar{q}_{2}^{2}}-\frac{4 b_{0}^{2} I_{p} \bar{q}_{2} x_{22}^{2}}{\left(b_{0}^{2}+\bar{q}_{2}^{2}\right)^{3}}\right) M_{a}\right] .
\end{aligned}
$$

Define the matrix $g_{2}\left(x_{1}, x_{2}\right)$ as

$$
g_{2}\left(x_{1}, x_{2}\right)=\left[\begin{array}{lll}
g_{211} & g_{212} & g_{213} \\
g_{221} & g_{222} & g_{223}
\end{array}\right] \text {. }
$$


According to equations (3.34) and (3.35), we have each component of the matrix as

$$
\begin{aligned}
g_{211} & =\frac{1}{\zeta_{4} \zeta_{1}}\left[\left(1-\frac{\zeta_{3}}{\zeta_{2}}\right)\left(\frac{1}{m_{1}}\right)\right] \\
g_{212} & =\frac{1}{\zeta_{4} \zeta_{1}}\left[\left(1-\frac{\zeta_{3}}{\zeta_{2}}\right)\left(\frac{1}{m_{1}+m_{2}}\right)\right] \\
g_{213} & =\frac{1}{\zeta_{4} \zeta_{1}}\left[\left(1+\frac{\zeta_{3}}{\zeta_{2}}\right)\left(\frac{1}{m_{3}}\right)\right] \\
g_{221} & =\left(\frac{1}{\zeta_{2}}-\frac{\zeta_{5}}{\zeta_{4} \zeta_{1} \zeta_{2}}\left(1-\frac{\zeta_{3}}{\zeta_{2}}\right)\right)\left(\frac{1}{m_{1}}\right) \\
g_{222} & =\left(\frac{1}{\zeta_{2}}-\frac{\zeta_{5}}{\zeta_{4} \zeta_{1} \zeta_{2}}\left(1-\frac{\zeta_{3}}{\zeta_{2}}\right)\right)\left(\frac{1}{m_{1}+m_{2}}\right) \\
g_{223} & =\left(-\frac{1}{\zeta_{2}}-\frac{\zeta_{5}}{\zeta_{4} \zeta_{1} \zeta_{2}}\left(1+\frac{\zeta_{3}}{\zeta_{2}}\right)\right)\left(\frac{1}{m_{3}}\right) .
\end{aligned}
$$

In this way, we have derived the state space form of the dynamic model of the cooperative manipulator robotic system with actuator redundancy, which can be used to design an adaptive actuator failure compensation scheme. 


\subsection{Nominal Control Design}

In this section we develop a nominal controller for the system assuming the knowledge of the actuator failures, such a controller structure can be used to design an adaptive actuator failure compensation scheme for the system with uncertain actuator failures. From the dynamic model derived in Section III, we have

$$
\begin{aligned}
& \dot{x}_{1}=f\left(x_{1}\right) x_{2} \\
& \dot{x}_{2}=g_{1}\left(x_{1}, x_{2}\right)+g_{2}\left(x_{1}, x_{2}\right) u \\
& y=x_{1},
\end{aligned}
$$

where $y=[h, \theta]^{T}$ is the system output, and $f\left(x_{1}\right), g_{1}\left(x_{1}, x_{2}\right), g_{2}\left(x_{1}, x_{2}\right)$ are known matrices.

Our goal is to control the trajectory of the height and angle of the platform; that is, we need to design a control algorithm such that the system output $y(t)$ tracks a desirable trajectory $y_{m}(t)$ asymptotically. First, we generate a desirable feedback control signal $\omega_{d}=g_{2}\left(x_{1}, x_{2}\right) u$ from backstepping control design method, and then we will develop a nominal failure compensation controller based on the feedback control signal.

\subsubsection{Backsteping Control Design}

We will first choose a control signal $\omega_{d}$ such that it can guarantee the closed-loop signal boundedness and asymptotic output tracking of the system in the absence of actuator failures. 
Define $z_{1}=x_{1}-y_{m}, z_{2}=x_{2}-\beta$, where $\beta$ is a design function to be determined.

Step 1: From the state equation of the system, we can write $\dot{z}_{1}$ as

$$
\begin{aligned}
\dot{z}_{1} & =\dot{x}_{1}-\dot{y}_{m} \\
& =f\left(x_{1}\right) x_{2}-\dot{y}_{m} \\
& =f\left(x_{1}\right)\left(z_{2}-\beta\right)-\dot{y}_{m} .
\end{aligned}
$$

In order to choose $\beta$ to stablize the system, we consider a Lyapunov candidate function and its derivative

$$
\begin{aligned}
& V_{1}=\frac{1}{2} z_{1}^{T} z_{1} \\
& \dot{V}_{1}=z_{1}\left(f\left(x_{1}\right) z_{2}+f\left(x_{1}\right) \beta-\dot{y}_{m}\right) .
\end{aligned}
$$

With the design function

$$
\beta=f\left(x_{1}\right)^{-1}\left(-c_{1} z_{1}+\dot{y}_{m}\right),
$$

where $c_{1}$ is a positive constant. The derivative of the Lyapunov function becomes

$$
\dot{V}_{1}=-c_{1} z_{1}^{T} z_{1}+f\left(x_{1}\right) z_{1} z_{2} .
$$

We see that the term $-c_{1} z_{1}^{T} z_{1}$ is always negative. 
Step 2: Since $z_{2} \neq 0$, we continue using backstepping design by considering

$$
\begin{aligned}
\dot{z}_{2} & =\dot{x}_{2}-\dot{\beta} \\
& =g_{1}\left(x_{1}, x_{2}\right)+g_{2}\left(x_{1}, x_{2}\right) u-\dot{\beta},
\end{aligned}
$$

and the second Lyapunov function

$$
V_{2}=V_{1}+\frac{1}{2} z_{2}^{T} z_{2}
$$

The time-derivative of $V_{2}$ is

$$
\begin{aligned}
\dot{V}_{2} & =\dot{V}_{1}+z_{2}^{T} \dot{z}_{2} \\
& =-c_{1} z_{1}^{T} z_{1}+z_{2}^{T}\left(f\left(x_{1}\right) z_{1}+g_{1}\left(x_{1}, x_{2}\right)+\omega_{d}-\dot{\beta}\right)
\end{aligned}
$$

As a result, the signal $\omega_{d}$ is chosen as

$$
\begin{aligned}
\omega_{d} & =g_{2}\left(x_{1}, x_{2}\right) u \\
& =-g_{1}\left(x_{1}, x_{2}\right)-c_{2} z_{2}-f\left(x_{1}\right) z_{1}+\dot{\beta} .
\end{aligned}
$$

With the chosen $\omega_{d}$, equation (3.65) becomes

$$
\dot{V}_{2}=-c_{1} z_{1}^{T} z_{1}-c_{2} z_{2}^{T} z_{2}
$$

With a positive constant $c_{2}, \dot{V}_{2}$ is negative semidefinite and only equal to zero when $z_{1}=z_{2}=0$. As a result, the control signal $\omega_{d}$ can guarantee the closed-loop signal boundedness and asymptotic output tracking of the system: $\lim _{t \rightarrow \infty}\left(y(t)-y_{m}(t)\right)=0$. 


\subsubsection{Nominal Controller Structure}

In this section, we develop a nominal controller for the system, with knowledge of actuator failure in the system. The nominal control law will guarantee that the control input signal matches the desirable signal $\omega_{d}$ when actuator failures occur.

Since the controller needs to handle several cases of actuator failure, we select a nominal controller structure as a combination of the nominal controller of each actuator failure case. We first design three individual control schemes for each actuator failure case.

\section{Design for no failure case}

In the case of no actuator failure in the system, we have control signal $u=v^{*}$. We need to design $v(t)$ such that $\omega_{d}=g_{2}\left(x_{1}, x_{2}\right) v^{*}$. We consider

$$
v^{*}(t)=v_{(1)}^{*}(t)=h_{a}\left(x_{1}, x_{2}\right) v_{a(1)}^{*}(t),
$$

where $h_{a}\left(x_{1}, x_{2}\right) \in R^{3 \times 3}$, and $v_{a(1)}^{*}(t) \in R^{3 \times 1}$ are such that

$$
g_{2}\left(x_{1}, x_{2}\right) h_{a}\left(x_{1}, x_{2}\right) v_{a(1)}^{*}=\omega_{d} .
$$

Notice that in case of no actuator failure, we have some flexibilities when choosing the matrix $h_{a}\left(x_{1}, x_{2}\right)$. We can choose the matrix such that the system is optimized in some perspectives. With chosen $h_{a}\left(x_{1}, x_{2}\right)$, the nominal control signal can be written as

$$
v_{a(1)}^{*}(t)=K_{a}\left(x_{1}, x_{2}\right) \omega_{d} .
$$


One way to design the controller to have a unique solution is to use only two actuators to control the system. By turning off actuator $u_{1}$, we can design the controller by choosing

$$
\begin{aligned}
v^{*}(t) & =v_{(1)}^{*}(t)=\left[\begin{array}{ll}
0 & v_{b(1)}^{* T}(t)
\end{array}\right]^{T} \\
v_{b(1)}^{*}(t) & =h_{1}\left(x_{1}, x_{2}\right) v_{0(1)}^{*}(t) .
\end{aligned}
$$

With $g_{2}=\left[g_{21}, g_{22}, g_{23}\right]=\left[g_{21}, g_{2(2)}\right]$, we can choose a new matrix $h_{1}\left(x_{1}, x_{2}\right) \in R^{2 \times 2}$ such that $g_{2(2)}\left(x_{1}, x_{2}\right) h_{1}\left(x_{1}, x_{2}\right)$ is invertible. Thus, $v_{0(1)}^{*}(t) \in R^{2 \times 1}$ can be chosen as

$$
v_{0(1)}^{*}(t)=K_{1}\left(x_{1}, x_{2}\right) \omega_{d},
$$

where the matrix $K_{1}\left(x_{1}, x_{2}\right) \in R^{2 \times 2}$ is expressed as

$$
K_{1}\left(x_{1}, x_{2}\right)=\left(g_{2(2)}\left(x_{1}, x_{2}\right) h_{1}\left(x_{1}, x_{2}\right)\right)^{-1} .
$$

As a result, we have the nominal control signal $v_{(1)}^{*}(t)$ that can be used to control the system with no actuator failure.

\section{Design for $u_{1}$ failure case}

In case of actuator $u_{1}$ fails, we have the signals $u_{1}=\bar{u}_{1}, u_{2}=v_{2}^{*}$, and $u_{3}=v_{3}^{*}$. The nominal control signal can be written as

$$
v^{*}(t)=v_{(2)}^{*}(t)=\left[\begin{array}{ll}
0 & v_{a(2)}^{* T}(t)
\end{array}\right]^{T} .
$$


With $g_{2}=\left[g_{21}, g_{22}, g_{23}\right]=\left[g_{21}, g_{2(2)}\right]$, we have

$$
v_{a(2)}^{*}(t)=h_{2}\left(x_{1}, x_{2}\right) v_{0(2)}^{*}(t),
$$

where the matrix $h_{2}\left(x_{1}, x_{2}\right) \in R^{2 \times 2}$ and the signal $v_{0(2)}^{*}(t) \in R^{2 \times 1}$ can be chosen to satisfy

$$
g_{21}\left(x_{1}, x_{2}\right) \bar{u}_{1}+g_{2(2)} h_{2}\left(x_{1}, x_{2}\right) v_{0(2)}^{*}(t)=\omega_{d}
$$

The signal $v_{0(2)}^{*}(t)$ can be written as

$$
v_{0(2)}^{*}(t)=K_{21}\left(x_{1}, x_{2}\right) \omega_{d}+K_{22}\left(x_{1}, x_{2}\right) \bar{u}_{1},
$$

where $K_{21} \in R^{2 \times 2}, K_{22} \in R^{2 \times 1}$ are expressed as

$$
\begin{aligned}
& K_{21}=\left(g_{2(2)} h_{2}\left(x_{1}, x_{2}\right)\right)^{-1} \\
& K_{22}=\left(g_{2(2)} h_{2}\left(x_{1}, x_{2}\right)\right)^{-1} g_{21}\left(x_{1}, x_{2}\right) .
\end{aligned}
$$

We can see that with this design, the nominal control signal $v_{(2)}^{*}(t)$ ensures $\omega_{d}=$ $g_{2}\left(x_{1}, x_{2}\right) v_{(2)}^{*}$ for the actuator $u_{1}$ failure case.

\section{Design for $u_{2}$ failure case}

Similarly, in case of actuator $u_{2}$ fails. The nominal control signal can be designed as

$$
v^{*}(t)=v_{(3)}^{*}(t)=\left[v_{1(3)}^{*}(t), 0, v_{3(3)}^{*}(t)\right]^{T} .
$$


Defining $v_{a(3)}^{*}(t) \in R^{2 \times 1}$ as

$$
v_{a(3)}^{*}(t)=\left[v_{1(3)}^{*}(t), v_{3(3)}^{*}(t)\right]^{T} .
$$

We design the control law from

$$
v_{a(3)}^{*}(t)=h_{3}\left(x_{1}, x_{2}\right) v_{0(3)}^{*}(t) .
$$

With $g_{2(1)}=\left[g_{21}, g_{23}\right]$, we can choose $h_{3}\left(x_{1}, x_{2}\right) \in R^{2 \times 2}$, and $v_{0(3)}^{*}(t) \in R^{2 \times 1}$ to satisfy

$$
g_{2(1)}\left(x_{1}, x_{2}\right) h_{3}\left(x_{1}, x_{2}\right) v_{0(3)}^{*}(t)+g_{22} \bar{u}_{2}=\omega_{d} .
$$

The signal $v_{0(3)}^{*}(t)$ can be written as

$$
\begin{aligned}
& v_{0(3)}^{*}(t)=K_{31}\left(x_{1}, x_{2}\right) \bar{u}_{2}+K_{32}\left(x_{1}, x_{2}\right) \omega_{d}, \\
& K_{31}=\left(g_{2(1)}\left(x_{1}, x_{2}\right) h_{3}\left(x_{1}, x_{2}\right)\right)^{-1} g_{22}\left(x_{1}, x_{2}\right) \\
& K_{32}=\left(g_{2(1)}\left(x_{1}, x_{2}\right) h_{3}\left(x_{1}, x_{2}\right)\right)^{-1} .
\end{aligned}
$$

We can see that the nominal control signal $v_{(3)}^{*}(t)$ ensures $\omega_{d}=g_{2}\left(x_{1}, x_{2}\right) v_{(3)}^{*}$ for the actuator $u_{2}$ failure case.

\section{Composite control design}

With the control structure for all three cases, we can design a composite control law for the system as

$$
v^{*}(t)=\rho_{1}^{*} v_{(1)}^{*}(t)+\rho_{2}^{*} v_{(2)}^{*}(t)+\rho_{3}^{*} v_{(3)}^{*}(t)
$$


where $\rho_{1}^{*}, \rho_{2}^{*}, \rho_{3}^{*}$ are the indicator functions of the actuator failure: $\rho_{1}^{*}=1$ when there is no actuator failure, $\rho_{2}^{*}=1$ for $u_{1}$ actuator failure, and $\rho_{3}^{*}=1$ for $u_{2}$ actuator failure. The indicator functions $\rho_{1}^{*}, \rho_{2}^{*}, \rho_{3}^{*}=0$ for its noncorresponding cases, e.g., $\rho_{1}^{*}=0$ when actuator $u_{1}$ fails. Signal $v_{(1)}^{*}(t)$ is the nominal control signal in case of no actuator failure in the system, $v_{(2)}^{*}(t)$ is the nominal control signal for the actuator $u_{1}$ failure case, and $v_{(3)}^{*}(t)$ is the nominal control signal for actuator $u_{2}$ failure case.

Because of the indicator functions and the nominal control signals, the composite design ensures $\omega_{d}=g_{2}\left(x_{1}, x_{2}\right) v^{*}(t)$ for every case of actuator failure. The composite control design can control the system with an actuator failure when the information of actuator failure is known. 


\subsection{Adaptive Failure Compensation Design}

The nominal control design, which is developed in Section IV, needs information of the actuator failures. In this section, we develop an adaptive actuator failure compensation scheme, which can achieve the control objective in the presence of uncertain actuator failures without the knowledge of the failing actuator and its signal.

\subsubsection{Adaptive Controller Structure}

The adaptive control algorithm is developed based on the nominal controller structure. Since we do not know the values of $\rho_{1}^{*} v_{(1)}^{*}, \rho_{2}^{*} v_{(2)}^{*}$, and $\rho_{3}^{*} v_{(3)}^{*}$, we first design the adaptive controller structure as

$$
v(t)=v_{\rho(1)}(t)+v_{\rho(2)}(t)+v_{\rho(3)}(t),
$$

where $v_{\rho(1)}(t), v_{\rho(2)}(t), v_{\rho(3)}(t)$ are the estimates of $\rho_{1}^{*} v_{(1)}^{*}, \rho_{2}^{*} v_{(2)}^{*}, \rho_{3}^{*} v_{(3)}^{*}$ respectively.

In order to derive $v_{\rho(1)}(t)$, we restructure $\rho_{1}^{*} v_{(1)}^{*} \in R^{3 \times 1}$ from the nonimal controller (3.69) as

$$
\begin{aligned}
\rho_{1}^{*} v_{(1)}^{*} & =\rho_{1}^{*} h_{a} K_{a} \omega_{d} \\
& =\operatorname{diag}\left\{\rho_{11}^{*}, \rho_{12}^{*}, \rho_{13}^{*}\right\} h_{a} K_{a} \omega_{d},
\end{aligned}
$$

where $\rho_{11}^{*}=\rho_{12}^{*}=\rho_{13}^{*}=\rho_{1}^{*}$; that is, we will estimate the parameter $\rho_{1}^{*}$ three times, which is needed for achieving a suitable parametrization. With the estimated param- 
eter $\operatorname{diag}\left\{\rho_{11}, \rho_{12}, \rho_{13}\right\}$, we have the adaptive version of $\rho_{1}^{*} v_{(1)}^{*}$ as

$$
v_{\rho(1)}(t)=\operatorname{diag}\left\{\rho_{11}, \rho_{12}, \rho_{13}\right\} h_{a} K_{a} \omega_{d} .
$$

With $v_{\rho(2)}(t)=\left[0, v_{a \rho(2)}(t)^{T}\right]^{T}$, we now derive $v_{a \rho(2)}(t)$ from its nominal version:

$$
\rho_{2}^{*} v_{a(2)}^{*}(t)=\rho_{2}^{*} h_{2} K_{21} \omega_{d}+\rho_{2}^{*} \bar{u}_{1} h_{2} K_{22} \in R^{2 \times 1} .
$$

From the actuator failure model (3.2), the actuator failure $\bar{u}_{i}(t)$ can be expressed as

$$
\bar{u}_{i}(t)=\chi_{i}^{* T} f_{a i}(t),
$$

where $f_{a i}(t)=\left[1, f_{a i 1}(t), \ldots, f_{a i n_{i}}(t)\right]^{T}$ are known functions corresponding to the actuator failure components, $\chi_{i}^{*}=\left[\bar{u}_{i 0}, \bar{u}_{i 1}, \ldots, \bar{u}_{i n_{i}}\right]^{T}$ contains the paremeters of values associated with each actuator failure component, and $n_{i}$ is the number of actuator failure components.

Then, the estimate of $\rho_{2}^{*} v_{a(2)}^{*}(t)$ is chosen as

$$
v_{\rho a(2)}(t)=\operatorname{diag}\left\{\rho_{21}, \rho_{22}\right\} h_{2} K_{21} \omega_{d}+\left[\begin{array}{c}
\chi_{1(1)}^{T} f_{a 1}(t) \phi_{11} \\
\chi_{1(2)}^{T} f_{a 1}(t) \phi_{12},
\end{array}\right]
$$

where, similar to that in (3.90), $\operatorname{diag}\left\{\rho_{21}, \rho_{22}\right\}$ is the estimate of $\operatorname{diag}\left\{\rho_{21}^{*}, \rho_{22}^{*}\right\}$ with $\rho_{2}^{*}=\rho_{21}^{*}=\rho_{22}^{*}$. The terms $\rho_{21}^{*} \chi_{1}^{*}$ and $\rho_{22}^{*} \chi_{1}^{*}$ are estimated by $\chi_{1(1)}$ and $\chi_{1(2)}$, where $\phi_{1}=\left[\phi_{11}, \phi_{12}\right]^{T}=h_{2} K_{22}$. 
In order to derive $v_{\rho(3)}$, we consider $v_{\rho(3)}=\left[v_{\rho(31)}, 0, v_{\rho(33)}\right]^{T}$. With $v_{a \rho(3)}=\left[v_{\rho(31)}, v_{\rho(33)}\right]^{T}$, we consider

$$
\rho_{3}^{*} v_{a(3)}^{*}(t)=\rho_{3}^{*} h_{3} K_{31} \bar{u}_{2}+\rho_{3}^{*} h_{3} K_{32} \omega_{d} \in R^{2 \times 1} .
$$

Similar to the derivation of $v_{\rho a(2)}(t)$, we have

$$
v_{\rho a(3)}(t)=\operatorname{diag}\left\{\rho_{31}, \rho_{32}\right\} h_{3} K_{32} \omega_{d}+\left[\begin{array}{c}
\chi_{2(1)}^{T} f_{a 2}(t) \phi_{21} \\
\chi_{2(2)}^{T} f_{a 2}(t) \phi_{22},
\end{array}\right],
$$

where $\operatorname{diag}\left\{\rho_{31}, \rho_{32}\right\}$ is the estimate of $\operatorname{diag}\left\{\rho_{31}^{*}, \rho_{32}^{*}\right\}$ with $\rho_{3}^{*}=\rho_{31}^{*}=\rho_{32}^{*}$. The parameters $\chi_{2(1)}$ and $\chi_{2(2)}$ are the estimates of $\rho_{31}^{*} \chi_{2}^{*}$ and $\rho_{32}^{*} \chi_{2}^{*}$, where $\phi_{2}=\left[\phi_{21}, \phi_{22}\right]^{T}=$ $h_{3} K_{31}$.

The controller structure (3.88) can be written as

$$
v(t)=v_{\rho(1)}(t)+\left[0, v_{a \rho(2)}^{T}(t)\right]^{T}+\left[v_{\rho(31)}(t), 0, v_{\rho(33)}(t)\right]^{T} .
$$

With this parametrized controller structure, we can develop adaptive laws to update the parameters $\operatorname{diag}\left\{\rho_{11}, \rho_{12}, \rho_{13}\right\}, \operatorname{diag}\left\{\rho_{21}, \rho_{22}\right\}, \operatorname{diag}\left\{\rho_{31}, \rho_{32}\right\}, \chi_{1(1)}, \chi_{1(2)}, \chi_{2(1)}$, and $\chi_{2(2)}$, so that the system can achieve the control objective in the presence of uncertain actuator failure.

\subsubsection{Error System}

In order to design an adaptive law for the system, we consider the system error caused by an actuator failure. With an uncertain actuator failure, the signal $u(t)$ can 
be written as

$$
u(t)=(I-\sigma(t)) v^{*}(t)+\sigma(t) \bar{u},
$$

where $\sigma(t)=\operatorname{diag}\left\{\sigma_{1}, \sigma_{2}, \sigma_{3}\right\}$ is the actuator failure pattern matrix such that $\sigma_{i}(t)=1$ if the $i$ actuator fails, and $\sigma_{i}(t)=0$ otherwise. With $g_{2}\left(x_{1}, x_{2}\right) u(t)=\omega_{d}$, we have

$$
g_{2}\left(x_{1}, x_{2}\right)\left((I-\sigma) v^{*}(t)+\sigma \bar{u}\right)=\omega_{d}
$$

For an adaptive updated control signal $v(t)$, where $g_{2}\left(x_{1}, x_{2}\right) v(t)=\omega$. Based on equations (3.97) and (3.98), we have an error system between $v(t)$ and $v^{*}(t)$ as

$$
g_{2}\left(x_{1}, x_{2}\right)(I-\sigma)\left(v-v^{*}\right)=\omega-\omega_{d} .
$$

We consider the backstepping design with a possible actuator failure. With $z_{1}=$ $x_{1}-y_{m}, z_{2}=x_{2}-\beta$, where $\beta$ is a design function from equation (3.60), we have

$$
\begin{aligned}
\dot{z}_{1} & =-c_{1} z_{1}+f\left(x_{1}\right) z_{2} \\
\dot{z}_{2} & =g_{1}\left(x_{1}, x_{2}\right)+g_{2}\left(x_{1}, x_{2}\right) u-\dot{\beta} \\
& =g_{1}\left(x_{1}, x_{2}\right)+\omega-\dot{\beta}+\left(\omega_{d}-\omega_{d}\right) \\
& =-f\left(x_{1}\right) z_{1}-c_{2} z_{2}+\omega-\omega_{d} .
\end{aligned}
$$

From equations (3.99) and (3.101), $\dot{z}_{2}$ becomes

$$
\dot{z}_{2}=-f\left(x_{1}\right) z_{1}-c_{2} z_{2}+g_{2}\left(x_{1}, x_{2}\right)(I-\sigma)\left(v-v^{*}\right) .
$$


Define the error signal $\tilde{v}=v-v^{*}$, and the error of each estimated parameter as $\tilde{\rho}_{i j}=\rho_{i j}-\rho_{i}^{*}, \tilde{\chi}_{i(j)}=\chi_{i(j)}-\left(\rho_{i+1 j}^{*} \chi_{i}^{*}\right)$. From equations (3.87) and (3.96), the error signal $\tilde{v}$ can be written as

$$
\begin{aligned}
\tilde{v} & =\operatorname{diag}\left\{\tilde{\rho}_{11}, \tilde{\rho}_{12}, \tilde{\rho}_{13}\right\} h_{a} K_{a} \omega_{d}+\left[0, \tilde{v}_{\rho a(2)}^{T}(t)\right]^{T} \\
& +\left[\tilde{v}_{\rho(31)}(t), 0, \tilde{v}_{\rho(33)}(t)\right]^{T}
\end{aligned}
$$

Based on equations (3.93) and (3.95) with $\tilde{v}_{\rho a(3)}(t)=\left[\tilde{v}_{\rho(31)}(t), \tilde{v}_{\rho(33)}(t)\right]^{T}$, we have

$$
\begin{aligned}
& \tilde{v}_{\rho a(2)}(t)=\operatorname{diag}\left\{\tilde{\rho}_{21}, \tilde{\rho}_{22}\right\} h_{2} K_{21}\left(x_{1}, x_{2}\right) \omega_{d}+\left[\begin{array}{c}
\tilde{\chi}_{1(1)}^{T} f_{a 1}(t) \phi_{11} \\
\tilde{\chi}_{1(2)}^{T} f_{a 1}(t) \phi_{12},
\end{array}\right] \\
& \tilde{v}_{\rho a(3)}(t)=\operatorname{diag}\left\{\tilde{\rho}_{31}, \tilde{\rho}_{32}\right\} h_{2} K_{32}\left(x_{1}, x_{2}\right) \omega_{d}+\left[\begin{array}{c}
\tilde{\chi}_{2(1)}^{T} f_{a 2}(t) \phi_{21} \\
\tilde{\chi}_{2(2)}^{T} f_{a 2}(t) \phi_{22},
\end{array}\right] .
\end{aligned}
$$

The effect of actuator failure to the nominal controller can be analyzed using the Lyapunov function

$$
V_{2}=\frac{1}{2}\left(z_{1}^{T} z_{1}+z_{2}^{T} z_{2}\right) .
$$

Based on equation (3.100) and (3.102), the derivative of the Lyapunov function (3.105) becomes

$$
\begin{aligned}
\dot{V}_{2}= & -c_{1} z_{1}^{T} z_{1}-c_{2} z_{2}^{T} z_{2} \\
& +z_{2} g_{2}\left(x_{1}, x_{2}\right)(I-\sigma)(\tilde{v}) .
\end{aligned}
$$


With $\tilde{v}$ from equation (3.103), the derivative of the Lyapunov function can be written as

$$
\begin{aligned}
& \dot{V}_{2}=-c_{1} z_{1}^{T} z_{1}-c_{2} z_{2}^{T} z_{2} \\
& +z_{2}^{T} g_{2}\left(x_{1}, x_{2}\right)(I-\sigma) \operatorname{diag}\left\{\tilde{\rho}_{11}, \tilde{\rho}_{12}, \tilde{\rho}_{13}\right\} h_{a} K_{a} \omega_{d} \\
& +z_{2}^{T} g_{2}\left(x_{1}, x_{2}\right)(I-\sigma)\left[0, \tilde{v}_{\rho a(2)}^{T}(t)\right]^{T} \\
& +z_{2}^{T} g_{2}\left(x_{1}, x_{2}\right)(I-\sigma)\left[\tilde{v}_{\rho(31)}(t), 0, \tilde{v}_{\rho(33)}(t)\right]^{T} .
\end{aligned}
$$

With the possible actuator failure, $\dot{V}_{2}$ is no longer negative semidefinite; thus, we cannot guarantee system stability. We need to design an adaptive scheme to update the parameters $\operatorname{diag}\left\{\rho_{11}, \rho_{12}, \rho_{13}\right\}, \operatorname{diag}\left\{\rho_{21}, \rho_{22}\right\}, \operatorname{diag}\left\{\rho_{31}, \rho_{32}\right\}, \chi_{1(1)}, \chi_{1(2)}, \chi_{2(1)}$, and $\chi_{2(2)}$; then, new Lyapunov functions will be used to ensure system stability.

\subsubsection{Adaptive Laws}

In this section, we develop an adaptive scheme for updating the parameter of the controller (3.88). The adaptive laws for $\operatorname{diag}\left\{\rho_{11}, \rho_{12}, \rho_{13}\right\}, \operatorname{diag}\left\{\rho_{21}, \rho_{22}\right\}$, and $\operatorname{diag}\left\{\rho_{31}, \rho_{32}\right\}$ can be generically chosen as

$$
\begin{aligned}
& \dot{\rho}_{1 j}=-\gamma_{1 j} z_{2}^{T} g_{2 j} \mu_{1 j} \\
& \dot{\rho}_{2 j}=-\gamma_{2 j} z_{2}^{T} g_{2(j+1)} \mu_{2 j} \\
& \dot{\rho}_{31}=-\gamma_{31} z_{2}^{T} g_{21} \mu_{31} \\
& \dot{\rho}_{32}=-\gamma_{33} z_{2}^{T} g_{23} \mu_{32},
\end{aligned}
$$


where $\gamma_{i j}>0$ is the adaptive gain, and

$$
\begin{aligned}
& \mu_{1}=\left[\mu_{11}, \mu_{12}, \mu_{13}\right]^{T}=h_{a} K_{a} \omega_{d} \\
& \mu_{2}=\left[\mu_{21}, \mu_{22}\right]^{T}=h_{2} K_{21} \omega_{d} \\
& \mu_{3}=\left[\mu_{31}, \mu_{32}\right]^{T}=h_{3} K_{31} \omega_{d},
\end{aligned}
$$

with $h_{a}, h_{2}, h_{3}, K_{a}, K_{21}$, and $K_{31}$ being the design matrices from the adaptive controller structure (3.89), (3.93), and (3.95).

Similarly, the adaptive laws for $\chi_{1(1)}, \chi_{1(2)}, \chi_{2(1)}$, and $\chi_{2(2)}$ can be chosen as

$$
\begin{aligned}
& \dot{\chi}_{1(j)}=-\Gamma_{(1 j)} f_{a j} z_{2}^{T} g_{2(j+1)} \phi_{1 j} \\
& \dot{\chi}_{2(1)}=-\Gamma_{(21)} f_{a 1} z_{2}^{T} g_{21} \phi_{21} \\
& \dot{\chi}_{2(2)}=-\Gamma_{(22)} f_{a 2} z_{2}^{T} g_{23} \phi_{22},
\end{aligned}
$$

where the parameter $\Gamma_{(i j)}=\Gamma_{(i j)}^{T}>0$ is the adaptive gain matrix. The function $\phi_{i}$ is defined as $\phi_{1}=\left[\phi_{11}, \phi_{12}\right]^{T}=h_{2} K_{22}$, and $\phi_{2}=\left[\phi_{21}, \phi_{22}\right]^{T}=h_{3} K_{31}$.

We then use a parameter projection scheme to ensure the boundedness of the estimated parameters in the presence of actuator failure uncertainties. We first consider the physical range of the indicator functions $\rho_{1}^{*}, \rho_{2}^{*}$, and $\rho_{3}^{*}$. From the definition of the indicator functions, we have

$$
0 \leq \rho_{1}^{*} \leq 1, \quad 0 \leq \rho_{2}^{*} \leq 1, \quad 0 \leq \rho_{3}^{*} \leq 1
$$

Because $\rho_{11}^{*}=\rho_{12}^{*}=\rho_{13}^{*}=\rho_{1}^{*}, \rho_{21}^{*}=\rho_{22}^{*}=\rho_{2}^{*}$, and $\rho_{31}^{*}=\rho_{32}^{*}=\rho_{3}^{*}$, we have $0 \leq \rho_{i j}^{*} \leq 1$. We can see that the estimated parameters $\rho_{i j}$ should also have the lower 
bound at 0 and the upper bound at 1 .

Let $\chi_{i k}^{* a}, \chi_{i k}^{* b}$ be the upper and lower bounds of the components $\chi_{i k}^{*}$ of $\chi_{i}^{*}$ for $i=1,2$, and $k=1,2, \ldots, k_{n}$, that is,

$$
\chi_{1 k}^{* a} \leq \chi_{1 k}^{*} \leq \chi_{1 k}^{* b}, \quad \chi_{2 k}^{* a} \leq \chi_{2 k}^{*} \leq \chi_{2 k}^{* b} .
$$

The upper and lower bounds $\chi_{i k}^{* a}, \chi_{i k}^{* b}$ can be obtained from the maximun and minimum values that each failing actuator can produce based on the actuator failure model (2).

With the upper and lower bounds of each component of $\chi_{i}^{*}$, we can see that each component of the estimated parameters $\chi_{i(j)}$ should also has the same boundaries as the component of $\chi_{i}$ because $\chi_{i(j)}$ is the estimate of $\chi_{i} \rho_{j}$ with $0 \leq \rho_{j} \leq 1$.

With the upper and lower bounds of the true parameters, we can modify the adaptive laws in (3.108) and (3.110), so that each component of the estimated parameters stays within its boundaries; and in particular, the initial conditions $\rho_{i j}(0), \chi_{i(j)}(0)$ are chosen inside the boundaries.

The adaptive laws for $\rho_{i j}(t)$ become

$$
\begin{aligned}
& \dot{\rho}_{1 j}=-\gamma_{1 j} z_{2}^{T} g_{2 j} \mu_{1 j}+\varrho_{\rho_{1} j} \\
& \dot{\rho}_{2 j}=-\gamma_{2 j} z_{2}^{T} g_{2(j+1)} \mu_{2 j}+\varrho_{\rho_{2} j} \\
& \dot{\rho}_{31}=-\gamma_{31} z_{2}^{T} g_{21} \mu_{31}+\varrho_{\rho_{3} 1} \\
& \dot{\rho}_{32}=-\gamma_{33} z_{2}^{T} g_{23} \mu_{32}+\varrho_{\rho_{3} 2},
\end{aligned}
$$


where the projection function $\varrho_{\rho i j}$ is chosen as

$$
\varrho_{\rho i j}=\left\{\begin{array}{ccc}
0 & \text { if } & \rho_{i j} \in(0,1), \\
& \text { or } \quad \rho_{i j}=0 & \text { and } \quad \varrho_{\rho i j} \geq 0, \\
& \text { or } \quad \rho_{i j}=1 \quad \text { and } \quad \varrho_{\rho i j} \leq 0, \\
-\bar{\varrho}_{\rho i j} & & \text { otherwise, }
\end{array}\right.
$$

with $\bar{\varrho}_{\rho i j}$ defined as

$$
\begin{aligned}
& \bar{\varrho}_{\rho 1 j}=-\gamma_{1 j} z_{2}^{T} g_{2 j} \mu_{1 j} \\
& \bar{\varrho}_{\rho 2 j}=-\gamma_{2 j} z_{2}^{T} g_{2(j+1)} \mu_{2 j} \\
& \bar{\varrho}_{\rho 31}=-\gamma_{31} z_{2}^{T} g_{21} \mu_{31} \\
& \bar{\varrho}_{\rho 32}=-\gamma_{33} z_{2}^{T} g_{23} \mu_{32} .
\end{aligned}
$$

Similarly, the adaptive laws for $\chi_{i(j)}=\left[\chi_{i(j)(1)}, \ldots, \chi_{i(j)\left(n_{i}\right)}\right]^{T}$ become

$$
\begin{aligned}
& \dot{\chi}_{1(j)}=-\Gamma_{(1 j)} f_{a j} z_{2}^{T} g_{2(j+1)} \phi_{1 j}+\varrho_{\chi_{1(j)}} \\
& \dot{\chi}_{2(1)}=-\Gamma_{(21)} f_{a 1} z_{2}^{T} g_{21} \phi_{21}+\varrho_{\chi_{2(1)}} \\
& \dot{\chi}_{2(2)}=-\Gamma_{(22)} f_{a 2} z_{2}^{T} g_{23} \phi_{22}+\varrho_{\chi_{2(2)}},
\end{aligned}
$$

where the projection function $\varrho_{\chi i(j)}=\left[\varrho_{\chi i(j)(1)}, \ldots, \varrho_{\chi i(j)\left(n_{i}\right)}\right]^{T}$ is chosen as

$$
\varrho_{\chi_{i(j)(k)}}=\left\{\begin{array}{ccc}
0 & \text { if } & \chi_{i(j)(k)} \in\left(\chi_{i k}^{* a}, \chi_{i k}^{* b}\right), \\
& \text { if } \quad \chi_{i(j)(k)}=\chi_{i k}^{* a} \text { and } \varrho_{\chi i(j)(k)} \geq 0, \\
& \text { or } \quad \chi_{i(j)(k)}=\chi_{i k}^{* b} \text { and } \varrho_{\chi i(j)(k)} \leq 0, \\
-\bar{\varrho}_{\chi i(j)(k)} & & \text { otherwise }
\end{array}\right.
$$


with $\bar{\varrho}_{\chi i(j)}=\left[\bar{\varrho}_{\chi i(j)(1)}, \ldots, \bar{\varrho}_{\chi i(j)\left(n_{i}\right)}\right]^{T}$ defined as

$$
\begin{aligned}
& \bar{\varrho}_{\chi 1(j)}=-\Gamma_{(1 j)} f_{a j} z_{2}^{T} g_{2(j+1)} \phi_{1 j} \\
& \bar{\varrho}_{\chi 2(1)}=-\Gamma_{(21)} f_{a 1} z_{2}^{T} g_{21} \phi_{21} \\
& \bar{\varrho}_{\chi 2(2)}=-\Gamma_{(22)} f_{a 2} z_{2}^{T} g_{23} \phi_{22} .
\end{aligned}
$$

It can be verified that the parameter projection schemes have the properties

$$
\begin{aligned}
\left(\rho_{i j}-\rho_{i j}^{*}\right) \varrho_{\rho_{i j}} & \leq 0 \\
\left(\chi_{i(j k)}-\rho_{i j}^{*} \chi_{i k}^{*}\right) \varrho_{\chi_{i(j k)}} & \leq 0,
\end{aligned}
$$

With the parameter projection scheme and chosen initial conditions $0 \leq \rho_{i j}(0) \leq$ $1, \chi_{i k}^{* a} \leq \chi_{i(j k)}(0) \leq \chi_{i k}^{* b}$, we can guarantee the boundedness of every estimated parameter used in the adaptive actuator failure compensation scheme.

\subsubsection{Performance Analysis}

The adaptive actuator failure compensation scheme can ensure the stability and tracking of the system in any of the three cases of possible actuator failure patterns: no failure occurs, actuator $u_{1}$ failure case, or actuator $u_{2}$ failure case, as shown by the following theorem.

Theorem 1. The adaptive actuator failure compensation scheme with the feedback control law (3.88) updated by the adaptive laws (3.113)-(3.116), when applied to the robotic model (3.52)-(3.54), guarantees the closed-loop signal boundedness and asymptotic output tracking: $\lim _{t \rightarrow \infty}\left(y(t)-y_{m}(t)\right)=0$. 
Proof. We first consider the error signals $z_{1}=x_{1}-y_{m}$, and $z_{2}=x_{2}-\beta$. With uncertain actuator failures, we have the derivatives of $z_{1}$ and $z_{2}$ as

$$
\begin{aligned}
\dot{z}_{1}= & -c_{1} z_{1}+f\left(x_{1}\right) z_{2} . \\
\dot{z}_{2}= & -f\left(x_{1}\right) z_{1}-c_{2} z_{2} \\
& +g_{2}\left(x_{1}, x_{2}\right)(I-\sigma)\left(v-v^{*}\right) .
\end{aligned}
$$

Because the derivative of the error signal $\dot{z}_{2}$ contains the actuator failure pattern $\sigma$ which changes according to each actuator failure case, we need to consider three Lyapunov function candidates $V_{a 0}, V_{a 1}, V_{a 2}$ for analyzing each actuator failure pattern to ensure the stability of the system. We first consider the case of no actuator failure, with the Lyapunov function candidate

$$
\begin{aligned}
V_{a 0}= & \frac{1}{2} z_{1}^{T} z_{1}+\frac{1}{2} z_{2}^{T} z_{2}+\frac{1}{2} \sum_{i=1}^{3} \tilde{\rho}_{1 i}^{2} \gamma_{1 i}^{-1} \\
& +\frac{1}{2}\left[\sum_{i=1}^{2} \tilde{\rho}_{2 i}^{2} \gamma_{2 i}^{-1}+\sum_{i=1}^{2} \tilde{\rho}_{3 i}^{2} \gamma_{3 i}^{-1}\right] \\
& +\frac{1}{2}\left[\sum_{i=1}^{2} \tilde{\chi}_{1(i)}^{T} \Gamma_{1 i}^{-1} \tilde{\chi}_{1(i)}+\sum_{i=1}^{2} \tilde{\chi}_{2(i)}^{T} \Gamma_{2 i}^{-1} \tilde{\chi}_{2(i)}\right] .
\end{aligned}
$$

With the parameter projection scheme (3.119) and (3.120), we have the derivative of each term in (3.122) as

$$
\begin{aligned}
\frac{d}{d t} & \frac{1}{2}\left(z_{1}^{T} z_{1}+z_{2}^{T} z_{2}\right)=-c_{1} z_{1}^{T} z_{1}-c_{2} z_{2}^{T} z_{2} \\
& +z_{2}^{T} g_{2}\left(x_{1}, x_{2}\right)(I-\sigma) \tilde{v}_{\rho(1)} \\
& +z_{2}^{T} g_{2}\left(x_{1}, x_{2}\right)(I-\sigma)\left[0, \tilde{v}_{\rho a(2)}^{T}(t)\right]^{T} \\
& +z_{2}^{T} g_{2}\left(x_{1}, x_{2}\right)(I-\sigma)\left[\tilde{v}_{\rho(31)}(t), 0, \tilde{v}_{\rho(33)}(t)\right]^{T}
\end{aligned}
$$




$$
\begin{aligned}
& \frac{d}{d t}\left(\frac{1}{2} \sum_{i=1}^{3} \tilde{\rho}_{1 i}^{2} \gamma_{1 i}^{-1}\right) \\
& \quad=-z_{2}^{T}\left[\sum_{i=1}^{3} \tilde{\rho}_{1 i} g_{2 i} \mu_{1 i}\right]+\sum_{i=1}^{3} \tilde{\rho}_{1 i} \gamma_{1 i}^{-1} \varrho_{\rho_{1 i}} \\
& \quad \leq-z_{2}^{T} g_{2}\left(x_{1}, x_{2}\right) \tilde{v}_{\rho(1)}
\end{aligned}
$$

$$
\begin{aligned}
\frac{d}{d t}( & \left.\frac{1}{2}\left(\sum_{i=1}^{2} \tilde{\rho}_{2 i}^{2} \gamma_{2 i}^{-1}+\sum_{i=1}^{2} \tilde{\chi}_{1(i)}^{T} \Gamma_{1 i}^{-1} \tilde{\chi}_{1(i)}\right)\right) \\
= & -z_{2}^{T} g_{2}\left(x_{1}, x_{2}\right)\left[0, \tilde{v}_{\rho a(2)}^{T}(t)\right]^{T} \\
& +\sum_{i=1}^{3} \tilde{\rho}_{2 i} \gamma_{2 i}^{-1} \varrho_{\rho_{2 i}}+\sum_{i=1}^{2} \tilde{\chi}_{1(i)}^{T} \Gamma_{1 i}^{-1} \varrho_{\chi_{1(i)}} \\
\leq & -z_{2}^{T} g_{2}\left(x_{1}, x_{2}\right)\left[0, \tilde{v}_{\rho a(2)}^{T}(t)\right]^{T}
\end{aligned}
$$

$$
\begin{aligned}
\frac{d}{d t}( & \left.\frac{1}{2}\left(\sum_{i=1}^{2} \tilde{\rho}_{3 i}^{2} \gamma_{3 i}^{-1}+\sum_{i=1}^{2} \tilde{\chi}_{2(i)}^{T} \Gamma_{2 i}^{-1} \tilde{\chi}_{2(i)}\right)\right) \\
= & -z_{2}^{T} g_{2}\left(x_{1}, x_{2}\right)\left[\tilde{v}_{\rho(31)}(t), 0, \tilde{v}_{\rho(33)}(t)\right]^{T} . \\
& +\sum_{i=1}^{3} \tilde{\rho}_{3 i} \gamma_{3 i}^{-1} \varrho_{\rho_{3 i}}+\sum_{i=1}^{2} \tilde{\chi}_{2(i)}^{T} \Gamma_{2 i}^{-1} \varrho_{\chi_{2(i)}} \\
\leq & -z_{2}^{T} g_{2}\left(x_{1}, x_{2}\right)\left[\tilde{v}_{\rho(31)}(t), 0, \tilde{v}_{\rho(33)}(t)\right]^{T} .
\end{aligned}
$$

Here we have used the parameter projection property (3.119) and (3.120); that is,

$$
\begin{aligned}
\tilde{\rho}_{i j} \gamma_{i j}^{-1} \varrho_{\rho_{i j}} & \leq 0 \\
\tilde{\chi}_{i(j)}^{T} \Gamma_{i j}^{-1} \varrho_{\chi_{i(j)}} & \leq 0 .
\end{aligned}
$$

Based on equations (3.123)-(3.126) with $\sigma=0$, the derivative of the Lyapunov function (3.122) becomes

$$
\dot{V}_{a 0} \leq-c_{1} z_{1}^{T} z_{1}-c_{2} z_{2}^{T} z_{2} \leq 0 .
$$


For the actuator $u_{1}$ failure case, we have the actuator failure pattern $\sigma=\operatorname{diag}\{1,0,0\}$. We need a new Lyapunov function candidate $V_{a 1}$ as

$$
\begin{aligned}
V_{a 1} & =\frac{1}{2} z_{1}^{T} z_{1}+\frac{1}{2} z_{2}^{T} z_{2}+\frac{1}{2}\left[\sum_{i=2}^{3} \tilde{\rho}_{1 i}^{2} \gamma_{1 i}^{-1}+\sum_{i=1}^{2} \tilde{\rho}_{2 i}^{2} \gamma_{2 i}^{-1}\right. \\
& \left.+\tilde{\rho}_{32}^{2} \gamma_{32}^{-1}+\sum_{i=1}^{2} \tilde{\chi}_{1(i)}^{T} \Gamma_{1 i}^{-1} \tilde{\chi}_{1(i)}+\tilde{\chi}_{2(2)}^{T} \Gamma_{22}^{-1} \tilde{\chi}_{2(2)}\right]
\end{aligned}
$$

To obtain the derivatives of the Lyapunov function candidates in (3.129), we consider the derivative of each term similar to the derivation in the case of no actuator failure. In this case, we have

$$
\begin{aligned}
\frac{d}{d t}( & \left.\frac{1}{2} \sum_{i=2}^{3} \tilde{\rho}_{1 i}^{2} \gamma_{1 i}^{-1}\right) \\
= & -z_{2}^{T} g_{2}\left(x_{1}, x_{2}\right)\left[0, \tilde{v}_{\rho a(2)}^{T}(t)\right]^{T}+\sum_{i=2}^{3} \tilde{\rho}_{2 i} \gamma_{2 i}^{-1} \varrho_{\rho_{2 i}} \\
\leq & -z_{2}^{T} g_{2}\left(x_{1}, x_{2}\right)(I-\sigma) \tilde{v}_{\rho(1)} \\
\frac{d}{d t}( & \left.\frac{1}{2}\left(\sum_{i=1}^{2} \tilde{\rho}_{2 i}^{2} \gamma_{2 i}^{-1}+\sum_{i=1}^{2} \tilde{\chi}_{1(i)}^{T} \Gamma_{1 i}^{-1} \tilde{\chi}_{1(i)}\right)\right) \\
= & -z_{2}^{T} g_{2}\left(x_{1}, x_{2}\right)\left[0, \tilde{v}_{\rho a(2)}^{T}(t)\right]^{T} \\
& +\sum_{i=1}^{2} \tilde{\rho}_{2 i} \gamma_{2 i}^{-1} \varrho_{\rho_{2 i}}+\sum_{i=1}^{2} \tilde{\chi}_{1(i)}^{T} \Gamma_{1 i}^{-1} \varrho_{\chi_{1(i)}} \\
\leq & -z_{2}^{T} g_{2}\left(x_{1}, x_{2}\right)(I-\sigma)\left[0, \tilde{v}_{\rho a(2)}^{T}(t)\right]^{T} \\
\frac{d}{d t}( & \left.\frac{1}{2}\left(\tilde{\rho}_{32}^{2} \gamma_{32}^{-1}+\tilde{\chi}_{2(2)} \Gamma_{22}^{-1} \tilde{\chi}_{2(2)}^{T}\right)\right) \\
= & -z_{2}^{T} g_{2}\left(x_{1}, x_{2}\right)\left[0, \tilde{v}_{\rho a(2)}^{T}(t)\right]^{T} \\
& +\tilde{\rho}_{32} \gamma_{32}^{-1} \varrho_{\rho_{32}}+\tilde{\chi}_{2(2)}^{T} \Gamma_{22}^{-1} \varrho_{\chi_{2(2)}} \\
\leq & -z_{2}^{T} g_{2}\left(x_{1}, x_{2}\right)(I-\sigma)\left[\tilde{v}_{\rho(31)}(t), 0, \tilde{v}_{\rho(33)}(t)\right]^{T} .
\end{aligned}
$$

We can notice that the terms $\tilde{\rho}_{11}^{2} \gamma_{11}^{-1}, \tilde{\rho}_{31}^{2} \gamma_{31}^{-1}$, and $\tilde{\chi}_{2(1)} \Gamma_{21}^{-1} \tilde{\chi}_{2(1)}$ disappear because $(I-\sigma)=\operatorname{diag}\{0,1,1\}$, with $\sigma=\operatorname{diag}\{1,0,0\}$. We can see that the terms in the 
derivatives of Lyapunov function candidates (3.130)-(3.132) cancel the non-negative semidefinite parts in (3.123), and the derivatives of the Lyapunov function (3.129) becomes

$$
\dot{V}_{a 1} \leq-c_{1} z_{1}^{T} z_{1}-c_{2} z_{2}^{T} z_{2} \leq 0 .
$$

For the actuator $u_{2}$ failure case, we have the actuator failure pattern $\sigma=\operatorname{diag}\{0,1,0\}$. We use a new Lyapunov function candidate

$$
\begin{aligned}
& V_{a 2}=\frac{1}{2} z_{1}^{T} z_{1}+\frac{1}{2} z_{2}^{T} z_{2}+\frac{1}{2}\left[\sum_{i=1,3} \tilde{\rho}_{1 i}^{2} \gamma_{1 i}^{-1}+\tilde{\rho}_{22}^{2} \gamma_{22}^{-1}\right. \\
& \left.+\sum_{i=1}^{2} \tilde{\rho}_{3 i}^{2} \gamma_{3 i}^{-1}+\tilde{\chi}_{1(2)}^{T} \Gamma_{12}^{-1} \tilde{\chi}_{1(2)}+\sum_{i=1}^{2} \tilde{\chi}_{2(i)}^{T} \Gamma_{2 i}^{-1} \tilde{\chi}_{2(i)}\right]
\end{aligned}
$$

With simular approach as actuator $u_{1}$ failure case, we can see that the terms $\tilde{\rho}_{12}^{2} \gamma_{12}^{-1}$, $\tilde{\rho}_{21}^{2} \gamma_{21}^{-1}$, and $\tilde{\chi}_{1(1)} \Gamma_{11}^{-1} \tilde{\chi}_{1(1)}$ disappear because $(I-\sigma)=\operatorname{diag}\{1,0,1\}$. In this case we have

$$
\dot{V}_{a 2} \leq-c_{1} z_{1}^{T} z_{1}-c_{2} z_{2}^{T} z_{2} \leq 0 .
$$

The derivatives of our Lyapunov functions (3.128), (3.133), and (3.135) for all actuator failure cases are negative semidefinite. With the parameter projection schemes, we can show that all signals in the system are bounded. Since $z_{1}(t), z_{2}(t), \dot{z}_{1}(t) \in$ $L^{\infty} \cap L^{2}$, based on the Barbalat lemma we can conclude that $\lim _{t \rightarrow \infty} z_{1}=0 . \nabla \nabla \nabla$

From Theorem 1, we can see that the adaptive actuator failure compensation scheme can guarantee the asymptotic tracking of the system subject to uncertain actuator failure for the system operating in any of the three actuator failure cases. 


\subsection{Simulations Study}

In this section we simulate the adaptive actuator failure compensation control scheme based on the robotic model subject to uncertain actuator failures.

\subsubsection{System Model and Simulation Conditions}

The simulations are performed based on the dynamic model of the robotic system $(3.38)-(3.40)$ as

$$
\begin{aligned}
& \dot{x}_{1}=f\left(x_{1}\right) x_{2} \\
& \dot{x}_{2}=g_{1}\left(x_{1}, x_{2}\right)+g_{2}\left(x_{1}, x_{2}\right) u \\
& y=x_{1}
\end{aligned}
$$

The matrix $f\left(x_{1}\right), g_{1}\left(x_{1}, x_{2}\right)$, and $g_{2}\left(x_{1}, x_{2}\right)$ are calculated from equation (3.42) (3.51), where the parameters $\zeta_{1}, \zeta_{2}, \zeta_{3}, \zeta_{4}$, and $\zeta_{5}$ are

$$
\begin{aligned}
& \zeta_{1}=7.25 \\
& \zeta_{2}=1+2.5 \cos ^{4} x_{12} \\
& \zeta_{3}=2.5 \cos ^{4} x_{12} \\
& \zeta_{4}=1+\frac{0.431}{\tan ^{4} x_{12}+2 \tan ^{2} x_{12}+1.75} \\
& \zeta_{5}=1.25 .
\end{aligned}
$$

The simulations assign the mass of each actuator as $m_{1}=m_{2}=m_{3}=1 \mathrm{~kg}$, the mass of the platform $m_{p}=10 \mathrm{~kg}$, the moment of inertia of the platform $I_{p}=1 \mathrm{~kg} \cdot \mathrm{m}^{2}$, and the length of the platform $b_{0}=1 \mathrm{~m}$.

In this study we consider three cases of actuator failure as follows: 
(i) No actuator failure case: $u(t)=v(t)$ for $0 \leq t<50 \mathrm{~s}$

(ii) Actuator $u_{2}$ failure case: $u_{2}=\bar{u}_{2}=5$ for $50 \leq t<100 \mathrm{~s}$

(iii) The failing actuator $u_{2}$ becomes normal again, no actuator failure case: $u(t)=$ $v(t)$ for $100 \leq t<150 s$

(iv) Actuator $u_{1}$ failure case: $u_{1}=\bar{u}_{1}=\sin (0.1 t)$ for $150 \leq t<200 \mathrm{~s}$

For simulation we choose $c_{1}=c_{2}=2, \gamma_{i j}=0.01, \Gamma_{i(j)}=0.01 I_{2}, f_{a i}=[1, \sin 0.1 t]^{T}$. The initial conditions are chosen as $y(0)=[1,1]^{T}, \rho_{i j}(0)=0$, and $\chi_{i(j)}=[0,0]^{T}$. The reference signal is chosen as $y_{m}(t)=[2+\sin (0.1 t), 0.5]^{T}$.

\subsubsection{Simulation Results}

The simulation results show the output of the system in Figure 3, the tracking error in Figure 4. The adaptive parameters are presented in Figure 5 - Figure 8. We can see that the tracking errors always go to zero for all three cases of actuator failures. 

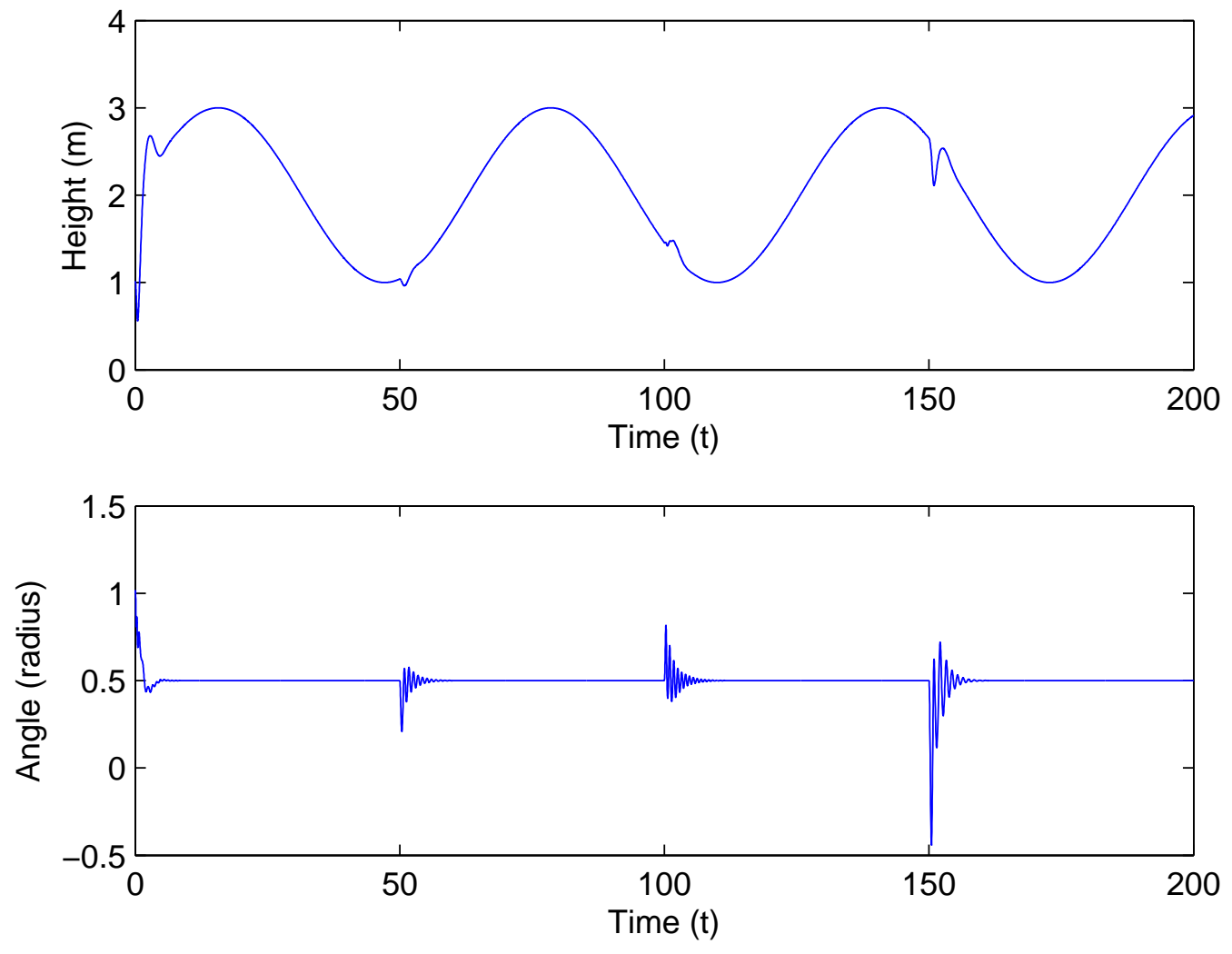

Figure 3.3: System outputs. 
CHAPTER 3. ADAPTIVE ACTUATOR FAILURE COMPENSATION FOR A
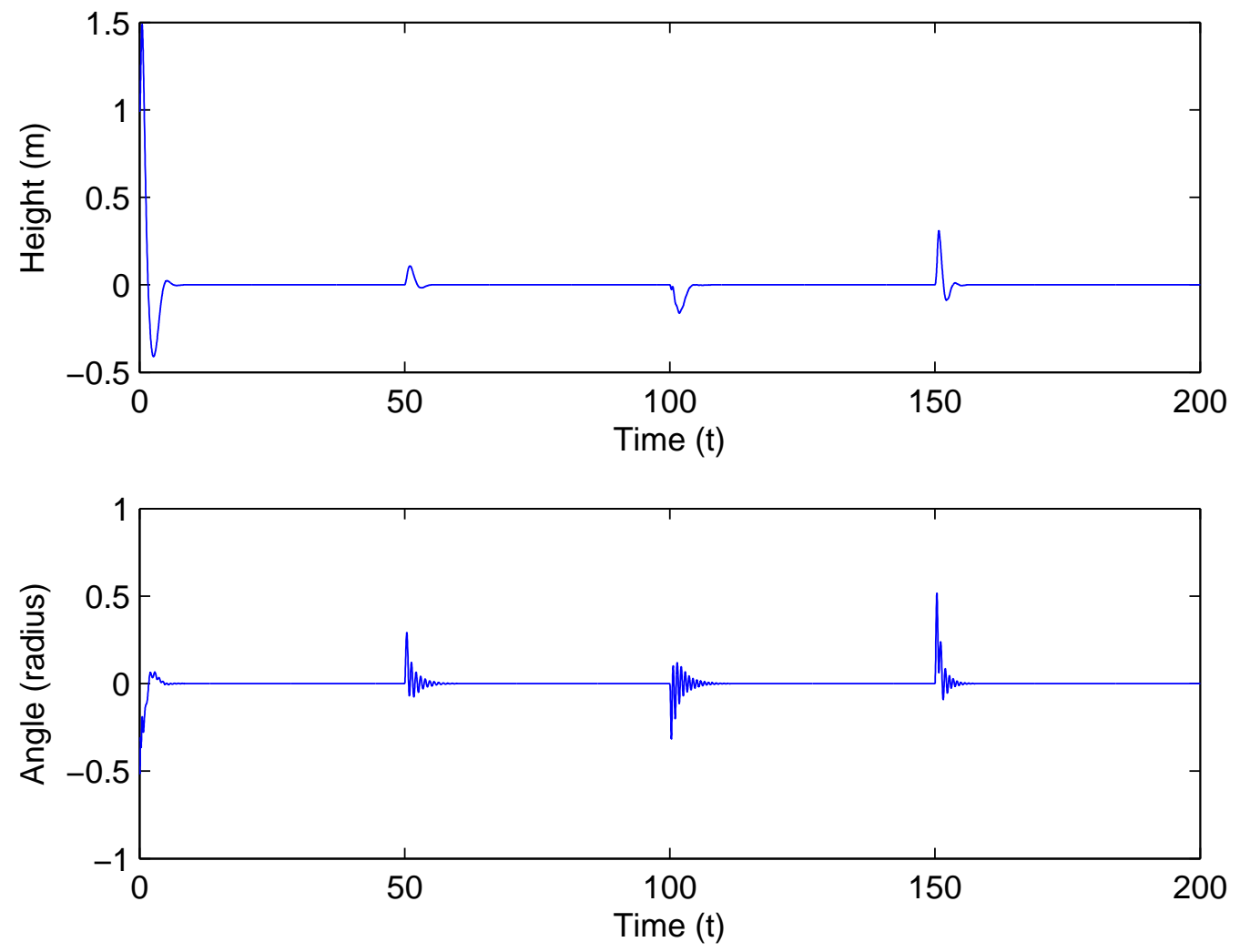

Figure 3.4: Tracking error $y_{m}(t)-y(t)$. 

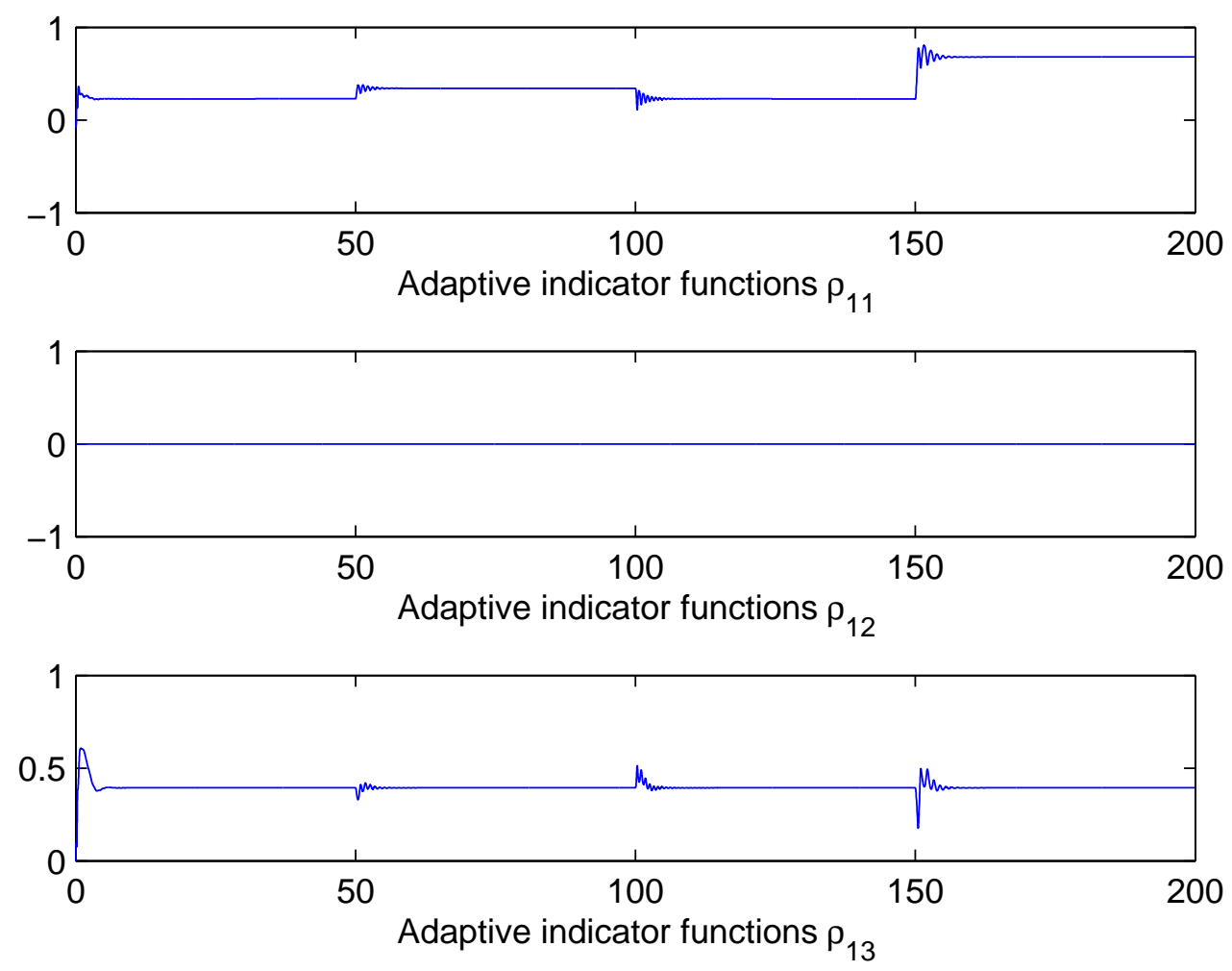

Figure 3.5: Adaptive indicator function $\rho_{11}, \rho_{12}, \rho_{13}$. 

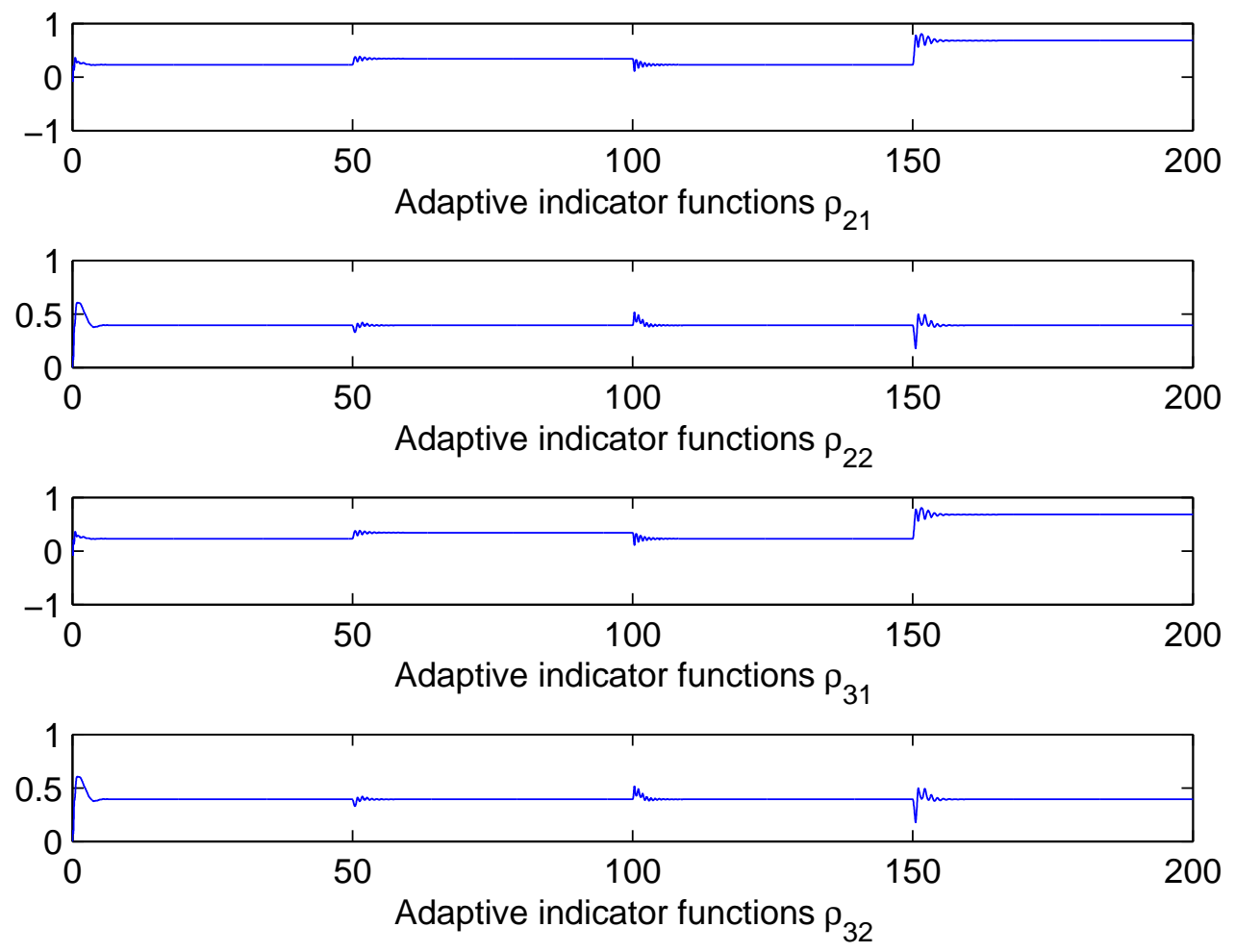

Figure 3.6: Adaptive indicator function $\rho_{21}, \rho_{22}, \rho_{31}, \rho_{32}$. 

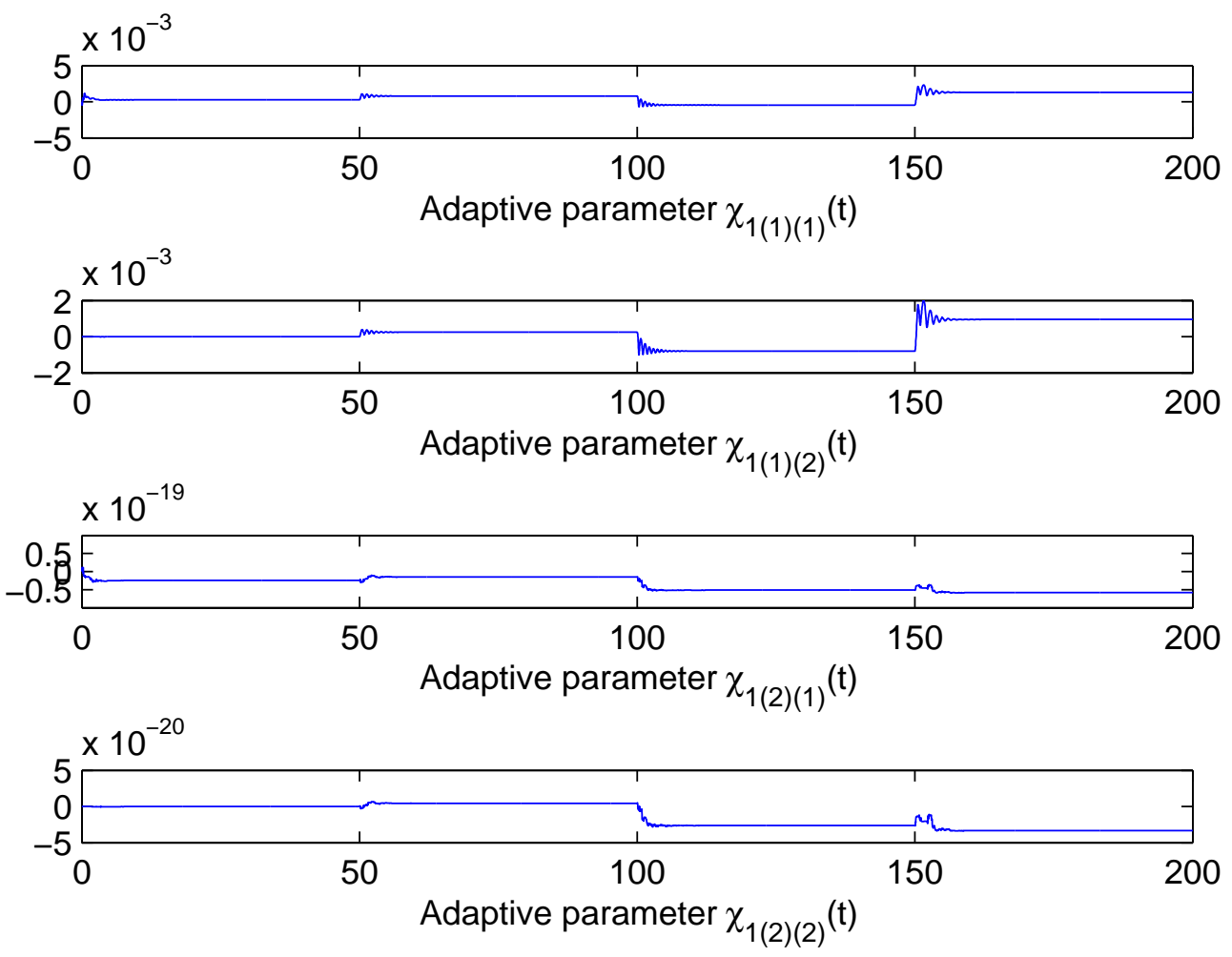

Figure 3.7: Adaptive parameters $\chi_{1(1)(1)}, \chi_{1(1)(2)}, \chi_{1(2)(1)}, \chi_{1(2)(2)}$. 

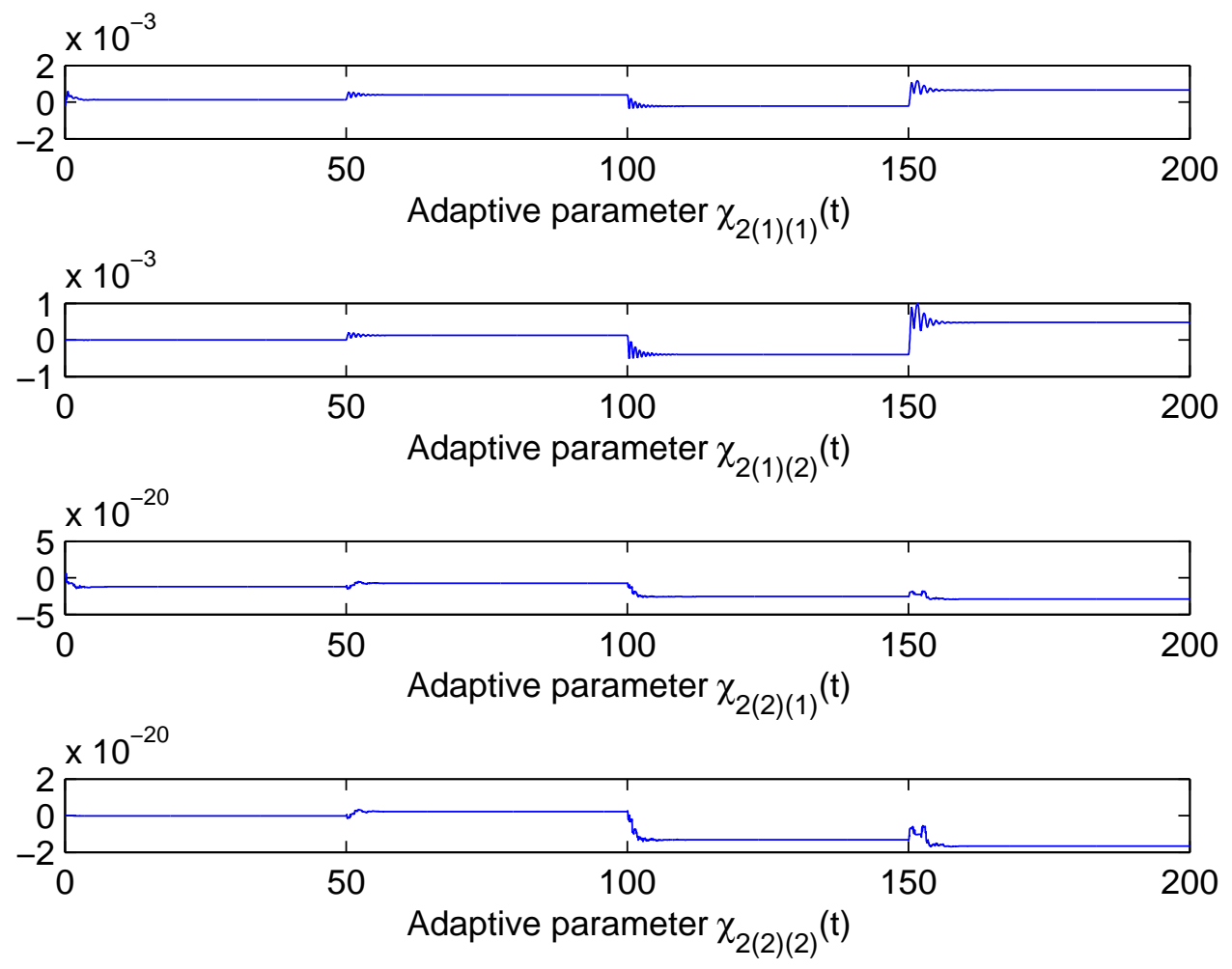

Figure 3.8: Adaptive parameters $\chi_{2(1)(1)}, \chi_{2(1)(2)}, \chi_{2(2)(1)}, \chi_{2(2)(2)}$. 
The simulation results confirm that the adaptive actuator failure compensation scheme can guarantee that the tracking error of the system goes to zero as time goes to infinity for a constant or a sinusoidal desirable trajectory. The adaptive actuator failure compensation scheme allows one actuator $u_{1}$ or $u_{2}$ to fail, but both actuators cannot fail at the same time. Finally, the simulation results show that adaptive actuator failure compensation scheme supports both a constant and a sinusoidal failure signal. 


\section{Chapter 4}

\section{Design Procedure for General Cases}

A cooperative manipulator robotic system uses multiple manipulators to control an object. Since the movement of the object depends on the coordination of every manipulator, an actuator failure in the system may compromise the entire system. It is important to develop an actuator failure compensation scheme for the system. Because a cooperative manipulator robotic system contains many manipulators, its often have enough redundancy which can be used in the adaptive actuator failure compensation scheme.

In this chapter, we discuss main ideas in developing an adaptive actuator failure compensation scheme for a general cooperative manipulator robotic system.

\subsection{General Dynamic Model}

The dynamic model of a cooperative manipulator robotic system can be divided into two parts, which are the dynamics of each manipulator and the dynamic of the object that controlled by the manipulators.

In order to derive the dynamic model of a general cooperative manipulator robotic system, we first consider the dynamic of each manipulator. Since each robotic manipulator in the system can be view as a rigid robot interacting with environment, 
we have the dynamic model of the $i$ th manipulator as [15]

$$
D_{i}\left(q_{i}\right) \ddot{q}_{i}+C_{i}\left(q_{i}, \dot{q}_{i}\right) \dot{q}_{i}+G_{i}\left(q_{i}\right)=\tau_{i}-J_{i}^{T} \hat{\tau}_{i},
$$

where $q_{i}$ is the joint angle of robot $i, \tau_{i}$ is the vector of joint torques, and $\hat{\tau}_{i}$ donates the interacting force between robot endeffector and the object. $D_{i}\left(q_{i}\right)$ is the inertia matrix, $C_{i}\left(q_{i}, \dot{q}_{i}\right)$ is the Coriolis and centrifugal term, $G_{i}\left(q_{i}\right)$ is the gravity term, and $J_{i}$ is the jacobian matrix from task space to Cartesian space.

We then obtain the dynamic model of the object. The general form of the dynamic model of a rigid object, which is interacting with the robotic manipulators, can be written as

$$
D_{o}\left(x_{o}\right) \ddot{x}_{o}+C_{o}\left(x_{o}, \dot{x}_{o}\right) \dot{x}_{o}+G_{o}\left(x_{o}\right)=\sum_{i=1}^{m} A_{i}^{T} \hat{\tau}_{i},
$$

where $x_{o}$ is the position of the object center of mass, $m$ is the number of the manipulators in the system, $D_{o}\left(x_{o}\right)$ is the inertia matrix of the object, $C_{o}\left(x_{o}, \dot{x}_{o}\right)$ is the Coriolis and centrifugal term of the object, $G_{o}\left(x_{o}\right)$ is the gravity term of the object, and $A_{i}$ is the augmented jacobian matrix of the object from the task space to Cartesian space.

Let $y_{i}$ be the coordinate of the endeffector of each manipulator, we have the relationship of each coordinate as

$$
\begin{aligned}
J_{i}\left(q_{i}\right) \dot{q}_{i} & =\dot{y}_{i} \\
A_{i}\left(x_{o}\right) \dot{x}_{o} & =\dot{y}_{i} .
\end{aligned}
$$

The general dynamic model of a cooperative manipulator robotic system can be obtained by combining (4.1) and (4.2) . With the coordinate transformation, the dynamic equation of the cooperative robotic system can be written in the task space 
as

$$
D_{c}\left(x_{o}\right) \ddot{x}_{o}+C_{c}\left(x_{o}, \dot{x}_{o}\right) \dot{x}_{o}+G_{c}\left(x_{o}\right)=E^{T}\left(x_{o}\right) \tau,
$$

where the combine inertia matrix of the object and manipulators $D_{c}\left(x_{o}\right)$ is assumed to be a bounded and positive definite matrix, $C_{c}\left(x_{o}, \dot{x}_{o}\right)$ is the Coriolis and centrifugal term, $G_{c}\left(x_{o}\right)$ is the combined gravity term, $E=\left[E_{1}^{T}, \ldots, E_{m}^{T}\right]^{T}$ with $E_{i}\left(x_{o}\right)=$ $J_{i}^{-1} A_{i}, i=1,2, \ldots, m$, and $\tau=\left[\tau_{1}^{T}, \ldots, \tau_{m}^{T}\right]^{T}, \tau_{i}$ is the generalized torque vector of the $i$ th manipulator.

With the general dynamic model of a cooperative manipulator system, we can design an adaptive actuator failure compensation scheme for the robotic system subject to uncertain actuator failures. 


\subsection{Adaptive Actuator Failure Compensation}

When an actuator failure occurs, the joint torque $\tau_{i}$ produced by that actuator becomes unknown. Because the complexity of the cooperative manipulator robotic system, it is difficult to identify failures in the system. In order to ensure the stability and tracking property of the system, we use the adaptive actuator failure compensation scheme developed in this study. We first rewrite the dynamic model in the state space form as

$$
\begin{aligned}
& \dot{x}_{1}=x_{2} \\
& \dot{x}_{2}=g_{1}\left(x_{1}, x_{2}\right)+g_{2}\left(x_{1}\right) u \\
& y=x_{1},
\end{aligned}
$$

where $y \in R^{n}$ is the output of the system, $x_{1}=x_{o} \in R^{n}, x_{2}=\dot{x}_{o} \in R^{n}$, and $u=\tau \in R^{m}$ is the input of the system. With the inertia matrix $D_{c}\left(x_{c}\right)$ nonsingular, we have

$$
\begin{aligned}
g_{1}\left(x_{1}, x_{2}\right) & =-D_{c}^{-1}\left(x_{1}\right)\left(C\left(x_{1}, x_{2}\right) x_{2}+G\left(x_{1}\right)\right) \in R^{n} \\
g_{2}\left(x_{1}\right) & =D_{c}^{-1}\left(x_{1}\right) E^{T}\left(x_{1}\right) \in R^{n \times m}
\end{aligned}
$$

where $\mathrm{n}$ is the degree of freedom of the system, and $\mathrm{m}$ is the number of actuator in the system.

\subsubsection{Nominal Control Design}

In order to develop an adaptive actuator failure compensation scheme for a general system subject to an unknown actuator failure, we need to develop a nominal con- 
troller structure similar to what we did in Section 3.3. We can generate a desirable feedback control signal $\omega_{d}=g_{2}\left(x_{1}, x_{2}\right) u \in R^{n}$ for general case from backstepping control design method.

Define $z_{1}=x_{1}-y_{m}, z_{2}=x_{2}-\beta$, where $y_{m} \in R^{n}$ is the desirable trajectory and $\beta=-c_{1} z_{1}+\dot{y}_{m}$. We have the desirable control signal

$$
\begin{aligned}
\omega_{d} & =g_{2}\left(x_{1}, x_{2}\right) u \\
& =-g_{1}\left(x_{1}, x_{2}\right)-c_{2} z_{2}-z_{1}+\dot{\beta},
\end{aligned}
$$

where $c_{1}, c_{2}$ are chosen to be some positive constants.

The nominal controller structure can be written as

$$
v^{*}(t)=\sum_{i=1}^{n_{p}} \rho_{i}^{*} v_{(i)}^{*}(t)
$$

where $\rho_{i}^{*}$ is the indicator functions associated with each actuator failure pattern, the signal $v_{(i)}^{*}(t)$ is the nominal control signal for each case of actuator failure in the system, and $n_{p}$ is the number of the actuator failure patterns that we consider.

The control signal $v_{(i)}^{*}(t)$ can be designed such that $\omega_{d}=g_{2}\left(x_{1}, x_{2}\right) v_{(i)}^{*}(t)$. For each case of actuator failure, we have

$$
v^{*}(t)=v_{(i)}^{*}(t)=A v_{a(i)}^{* T}(t),
$$

where $A \in R^{m \times n}$ is the actuator failure pattern matrix. The nominal control signal can be written as

$$
v_{a(i)}^{*}(t)=h_{i}\left(x_{1}, x_{2}\right) v_{0(i)}^{*}(t),
$$


where the matrix $h_{i}\left(x_{1}, x_{2}\right) \in R^{n \times n}$. The signal $v_{0(i)}^{*}(t) \in R^{n \times 1}$ can be chosen to satisfy

$$
g_{2(i) 1}\left(x_{1}, x_{2}\right) \bar{u}_{i}+g_{2(i) 2} h_{i}\left(x_{1}, x_{2}\right) v_{0(i)}^{*}(t)=\omega_{d},
$$

where $g_{2(i) 1}$ is a column of the matrix $g_{2}$ associated with the actuator failure pattern, and $g_{2(i) 2}$ is a part of the matrix $g_{2}$ without the column in $g_{2(i) 1}$.

The signal $v_{0(i)}^{*}(t)$ can be written as

$$
v_{0(i)}^{*}(t)=K_{i 1}\left(x_{1}, x_{2}\right) \omega_{d}+K_{i 2}\left(x_{1}, x_{2}\right) \bar{u}_{i},
$$

where $K_{i 1} \in R^{n \times n}, K_{i 2} \in R^{n \times 1}$ are expressed as

$$
\begin{aligned}
& K_{i 1}=\left(g_{2(i) 2} h_{i}\left(x_{1}, x_{2}\right)\right)^{-1} \\
& K_{i 2}=\left(g_{2(i) 2} h_{i}\left(x_{1}, x_{2}\right)\right)^{-1} g_{2(i) 1}\left(x_{1}, x_{2}\right) .
\end{aligned}
$$

We can see that with this design, the nominal control signal $v_{(i)}^{*}(t)$ ensures $\omega_{d}=$ $g_{2}\left(x_{1}, x_{2}\right) v_{(i)}^{*}$ for each actuator failure case.

\subsubsection{Adaptive Actuator Failure Compensation Design}

The nominal control design, which is developed in previous section, needs information of the actuator failures. In this section, we develop an adaptive actuator failure compensation scheme, which can achieve the control objective in the presence of an uncertain actuator failures, for a general class of robotic system.

From the nominal controller structure (4.10), we design the adaptive controller 
structure as

$$
v^{*}(t)=\sum_{i=1}^{n_{p}} v_{\rho(i)}(t),
$$

where $v_{\rho(i)}(t)$ is the estimate of $\rho_{i}^{*} v_{(i)}^{*}(t)$. In order to derive $v_{\rho(i)}(t)$, we consider

$$
v_{\rho(i)}(t)=A v_{a \rho(i)}(t),
$$

where $A \in R^{m \times n}$ is the actuator failure pattern matrix. We can derive $v_{a \rho(i)}(t)$ from it nominal version:

$$
\rho_{i}^{*} v_{a(i)}^{*}(t)=\rho_{i}^{*} h_{i} K_{i 1} \omega_{d}+\rho_{i}^{*} \bar{u}_{i} h_{i} K_{i 2} .
$$

With the actuator failure model

$$
\bar{u}_{j}(t)=\bar{u}_{j 0}+\sum_{i=1}^{n_{j}} \bar{u}_{i j} f_{a i j}(t), t \geq t_{j},
$$

we restructure $\rho_{i}^{*} v_{a(i)}^{*}(t)$ to obtain the estimate $v_{a \rho(i)}(t)$ as

$$
v_{\rho a(i)}(t)=\operatorname{diag}\left\{\rho_{i 1}, \rho_{i 2}, \ldots, \rho_{i n}\right\} h_{i} K_{i 2} \omega_{d}+\left[\begin{array}{c}
\tilde{\chi}_{i(1)}^{T} f_{a i}(t) \phi_{i 1} \\
\tilde{\chi}_{i(2)}^{T} f_{a i}(t) \phi_{i 2} \\
\vdots \\
\tilde{\chi}_{i(n)}^{T} f_{a i}(t) \phi_{i n}
\end{array}\right]
$$

The adaptive laws for updating the parameter $\rho_{i j}$ of the controller (4.16) can be generically chosen as

$$
\dot{\rho}_{i j}=-\gamma_{i j} z_{2}^{T} g_{2 j} \mu_{i j},
$$


where $\gamma_{i j}>0$ is the adaptive gain, and

$$
\mu_{i}=\left[\mu_{i 1}, \mu_{i 2}, \ldots, \mu_{i n}\right]^{T}=h_{i} K_{i 2} \omega_{d}
$$

with $h_{i}$, and $K_{i 2}$ being the design matrices from the adaptive controller structure (4.20).

Similarly, the adaptive laws for $\chi_{i(j)}$ can be chosen as

$$
\dot{\chi}_{i(j)}=-\Gamma_{(i j)} f_{a j} z_{2}^{T} g_{2 j} \phi_{i j}
$$

where the parameter $\Gamma_{(i j)}=\Gamma_{(i j)}^{T}>0$ is the adaptive gain matrix. The function $\phi_{i}$ is defined as $\phi_{i}=\left[\phi_{i 1}, \phi_{i 2}, \ldots, \phi_{i n}\right]^{T}=h_{i} K_{i 1}$.

In order to ensure the boundedness of the estimated parameters in the presence of actuator failure uncertainties, we consider using a parameter projection scheme. We first consider the physical range of the indicator functions $\rho_{i}^{*}$. From the definition of the indicator functions, we have $0 \leq \rho_{i}^{*} \leq 1$. Because $\rho_{i j}^{*}=\rho_{i}^{*}$ We can see that the estimated parameters $\rho_{i j}$ should also have the lower bound at 0 and the upper bound at 1 .

Let $\chi_{i j}^{* a}, \chi_{i j}^{* b}$ be the upper and lower bounds of the components $\chi_{i j}^{*}$ that is,

$$
\chi_{i j}^{* a} \leq \chi_{i j}^{*} \leq \chi_{i j}^{* b}
$$

With the upper and lower bounds of each component of $\chi_{i}$, we can see that each component of the estimated parameters $\chi_{i(j)}$ should also has the same boundaries as the component of $\chi_{i}$ because $\chi_{i(j)}$ is the estimate of $\chi_{i}^{*} \rho_{j}^{*}$ with $0 \leq \rho_{j}^{*} \leq 1$.

With the upper and lower bounds of the true parameters, we can modify the adaptive laws, so that each component of the estimated parameters stays within its 
boundaries; and in particular, the initial conditions $\rho_{i j}(0), \chi_{i(j)}(0)$ are chosen inside the boundaries.

The adaptive laws for $\rho_{i j}(t)$ becomes

$$
\dot{\rho}_{i j}=-\gamma_{i j} z_{2}^{T} g_{2 j} \mu_{i j}+\varrho_{\rho_{i} j},
$$

where the projection function $\varrho_{\rho i j}$ is chosen as

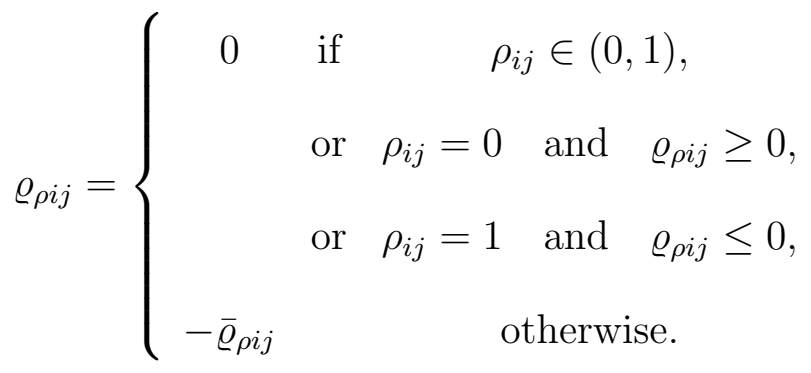

with $\bar{\varrho}_{\rho i j}$ is defined as

$$
\bar{\varrho}_{\rho i j}=-\gamma_{i j} z_{2}^{T} g_{2 j} \mu_{i j} .
$$

Similarly, the adaptive laws for $\chi_{i(j)}=\left[\chi_{i(j)(1)}, \ldots, \chi_{i(j)\left(n_{i}\right)}\right]^{T}$ becomes

$$
\dot{\chi}_{i(j)}=-\Gamma_{(i j)} f_{a j} z_{2}^{T} g_{2 j} \phi_{i j}+\varrho_{\chi_{1(j)}},
$$

where the projection function $\varrho_{\chi i(j)}=\left[\varrho_{\chi i(j)(1)}, \ldots, \varrho_{\chi i(j)\left(n_{i}\right)}\right]^{T}$ is chosen as

$$
\varrho_{\chi_{i(j)(k)}}=\left\{\begin{array}{ccc}
0 & \text { if } & \chi_{i(j)(k)} \in\left(\chi_{i k}^{* a}, \chi_{i k}^{* b}\right), \\
& \text { if } & \chi_{i(j)(k)}=\chi_{i k}^{* a} \text { and } \varrho_{\chi i(j)(k)} \geq 0, \\
& \text { or } & \chi_{i(j)(k)}=\chi_{i k}^{* b} \text { and } \varrho_{\chi i(j)(k)} \leq 0, \\
-\bar{\varrho}_{\chi i(j)(k)} & & \text { otherwise. }
\end{array}\right.
$$


with $\bar{\varrho}_{\chi i(j)}=\left[\bar{\varrho}_{\chi i(j)(1)}, \ldots, \bar{\varrho}_{\chi i(j)\left(n_{i}\right)}\right]^{T}$ is defined as

$$
\bar{\varrho}_{\chi 1(j)}=-\Gamma_{(i j)} f_{a j} z_{2}^{T} g_{2 j} \phi_{i j} .
$$

With the parameter projection scheme and chosen initial conditions $0 \leq \rho_{i j}(0) \leq$ $1, \chi_{i k}^{* a} \leq \chi_{i(j k)}(0) \leq \chi_{i k}^{* b}$, we can guarantee the boundedness of every estimated parameter used in the adaptive actuator failure compensation scheme.

It can be proven that the adaptive actuator failure compensation scheme can guarantee the asymptotic tracking of the system subject to uncertain actuator failure for the system operating in each actuator failure case.

\subsubsection{Design Procedure for General Case}

In summary, we can design an adaptive actuator failure compensation scheme by the following design procedure:

(i) Obtain a desirable signal $\omega_{d}=g_{2}\left(x_{1}\right) u$ by using the backstepping design method, which can guarantee the closed-loop signal boundedness and aspmtotic output tracking of the system in the absence of actuators failures.

(ii) Assuming all actuator failures are known, develop a nominal control for the system, which can produce a control signal $v$ such that $\omega_{d}=g_{2}\left(x_{1}\right) v$ for every desirable actuator failure pattern $\sigma$.

(iii) Derive the adaptive controller structure for the system subject to uncertain actuator failure with the controller parameterization based on the nominal controller structure developed in part (ii).

(iv) Develop an adaptive scheme with parameter projection for updating the estimated parameters used in the adaptive controller structure. 
Such an adaptive actuator failure compensation scheme is applicable to specific system under this general cooperative robotic system model to improve the performance of various cooperative manipulator robotic system subject to uncertain actuator failures. 


\section{Chapter 5}

\section{Conclusions and Future Work}

\subsection{Conclusions}

In this work we have developed a new adaptive actuator failure compensation scheme for a nonlinear multi-input multi-output cooperative robotic system subject to uncertain actuator failure. As shown in Chapter III of this thesis, the adaptive control scheme can ensure desirable closed-loop stability and asymptotic tracking of the system subject to three cases of actuator failures. The results show that the algorithm ensure the tracking of the system with both constant and sinusoidal desirable trajectory.

The adaptive actuator failure compensation scheme can guarantee desirable closedloop stability and asymptotic tracking without the knowledge of which actuator is failing and how much that actuator fails. If we know the output function that the failing actuator can produce, we can design an estimated parameter to compensate for such failure. From the simulation results we can also see that the algorithm can work with both actuator degradation case and complete failure case.

We also expand the knowledge of the adaptive actuator failure compensation scheme into a general class of cooperative manipulator robotic system. The research show that we can design an adaptive controller that can guarantee system stability 
and asymptotic tracking for a class of the system subject to unknown actuator failure without using an explicit fault identification scheme.

\subsection{Future Work}

In this study, we only consider using the torque of the motors as inputs of the system. For the next step of this study, we can consider adding dynamic model of the motor in the system. By consider using electrical voltage of motors as input of the system, we can develop a more practical adaptive control scheme that can be used in various robotic system.

In Chapter four, we only consider a simple class of cooperative manipulator robotic system. In the future we can study other type of the system in order to extend the application of the adaptive actuator failure compensation scheme to other classes of cooperative robotic system. For example, a cooperative robotic system interacting with geometrically unknown environment is an interesting application that we can study.

Another aspect that we could consider is how to improve the performance of the adaptive control algorithm that we had developed. One aspect might be to consider the effect of other type of uncertainty to the system such as parameter uncertainty or structure uncertainty, so that we can develop a more robust algorithm for the system. 


\section{Bibliography}

[1] M. Dede, S. Tosunoglu. "Fault-tolerant teleoperation systems design" Industrial Robot: An International Journal, Vol. 33/5, pp. 365372, 2006.

[2] K. Ohno, S. Kawatsuma, T. Okada, E. Takeuchi, K. Higashi, S. Tadokoro, "Robotic control vehicle for measuring radiation in Fukushima Daiichi Nuclear Power Plant ," Proceedings of the IEEE International Symposium on Safety, Security, and Rescue Robotics (SSRR), pp. 38 - 43, November, 2011.

[3] K. Jones, "Special Weapons Observation Remote recon Direct Action System (SWORDS)," In Platform Innovations and System Integration for Unmanned Air, Land and Sea Vehicles, pp. 36-1 36-8. 2007.

[4] S. Weinberger , "Armed Robots Still in Iraq, But Grounded," April 15, 2008. Available at http://www.wired.com/dangerroom/2008/04/armed-robots-st/.

[5] G. Liu, "Control of Robot Manipulators with Consideration of Actuator Performance Degradation and Failures" Proceedings of the 2001 IEEE International Conference on Robotics and Automation, Seoul, Korea. May 2001.

[6] M. Ozkan, G. Kirlik, O. Parlaktuna, A. Yufka and A. Yazici. "A Multi-Robot Control Architecture for Fault-Tolerant Sensor-Based Coverage" International Journal of Advanced Robotic Systems, Vol. 7, No. 1, pp. 067-074, 2010 
[7] A.H. Al-Bayati, H. Wang, "A Robust New Nonlinear Fault Tolerant Control for Robot via an Observer" Proceedings of the 8th World Congress on Intelligent Control and Automation, June 2011.

[8] M. Ji, N. Sarkar. "Supervisory Fault Adaptive Control of a Mobile Robot and Its Application in Sensor-Fault Accommodation" IEEE Transactions on Robotics, Vol. 23, No. 1, February, 2007.

[9] X. Zhang, M. M. Polycarpou, and T. Parisini, "Fault diagnosis of a class of nonlinear uncertain systems with Lipschitz nonlinearities using adaptive estimation, Automatica, vol 46, no. 2, 2010, pp. 290-299.

[10] Y. Diao, K. M. Passino, "Stable fault-tolerant adaptive fuzzy/neural control for turbine engine, IEEE Trans. on Control systems Technology, vol. 9, no. 3, 2001, pp. 494-509.

[11] B. Jiang, Z. Gao, P. Shi, Y. Xu, "Adaptive fault-tolerant tracking control of nearspace vehicle using takagi-sugeno fuzzy models, IEEE Trans. on fuzzy systems. vol. 18, no. 5, 2010, pp. 1000-1007.

[12] C. Axenie, D. Cernega, "Adaptive Sliding Mode Controller Design for Mobile Robot Fault Tolerant Control. Introducing ARTEMIC." 19th International Workshop on Robotics in Alpe-Adria-Danube Region RAAD 2010, Budapest, Hungary, June 2325, 2010.

[13] L. Wu, J. Lam and T. Parisini, "Sliding mode control of switched hybrid systems with time-varying delay, Internation Journal of Adaptive Control and Signal Processing, vol 22, no. 1, 2008, pp. 990-931.

[14] X. L. Yao, G. Tao, R. Y. Qi and B. Jiang, "An adaptive actuator failure compensation scheme for an attitude dynamic model of near space vehicles," Proceedings 
of the 2012 American Control Conference, pp. 368-373, Montreal, Canada, June 2012.

[15] Y. Lui, X. Tang, and G. Tao, "Adaptive failure compensation control of autonomous robotic systems: Application to a precision pointing hexapod," Proceedings of AIAA Infotech@Aerospace Conference, Arlington, VA, 2005.

[16] J. H. Jean, L. C. Fu, "An Adaptive Control Scheme for Coordinated Multimanipulator Systems," IEEE Transactions on Robotics and Automation, Vol. 9, No2, April 1993.

[17] F. Kececi, X. Tang, G. Tao "Adaptive actuator failure compensation for redundant manipulator" Robotica, Vol. 27, Issue 1. January, 2009.

[18] M.W. Spong and M. Vidyasagar, Robot Dynamics and Control, John Wiley \& Sons, New York, Inc., 1989.

[19] M.P. Lazarevic, Mathematical Modeling and Controlof Redundant Robotic Manipulators using Biological Analog, Mechanics Automatic Control and Robotics, Vol. 3, No 11, 201, pp. 285-294.

[20] X. Tang, G. Tao, S.M. Joshi, "Adaptive actuator failure compensation for nonlinear MIMO systems with an aircraft control application," Automatica, Vol 43, March 2007, pp. 1869-1883.

[21] M. Honegger, A. Codourey, E. Burdet, "Adaptive Control of the Hexaglide, a 6 dof Parallel Manipulator,” Proceedings of the 1997 IEEE International Conference on Robotics and Automation, Albuquerque, New Mexico, April 1997. 
[22] G. Yang, I.M. Chen, W.K. Lim, S.H. Yeo, "Design and Kinematic Analysis of Modular Reconfigurable Parallel Robots," Proceedings of the I999 IEEE International Conference on Robotics and Automation, Michigan, May 1999.

[23] H. Yang, B. Jiang, M. Staroswiecki, "Supervisory fault tolerant control for a class of uncertain nonlinear systems," Automatica, Vol 45, June 2009, pp. 2319-2324.

[24] S.S. Ge., J. Zhang, T.H. Lee, "Adaptive MNN control for a class of non-affine NARMAX systems with disturbances," Systems and Control Letters, Vol 53, 2004, pp. 1-12.

[25] G. Paviglianiti, F. Pierri, F. Caccavale, M. Mattei, "Robust fault detection and isolation for proprioceptive sensors of robot manipulators," Mechatronics, Vol. 20, 2010, pp. 162-170.

[26] B. Jiang, M. Staroswiecki, V. Cocquempot, "Fault Accommodation for Nonlinear Dynamic Systems," IEEE Transections on Automatic Control, Vol. 51, No. 9, September 2006, pp. 1587-1583.

[27] S.N. Huang, K.K. Tan, "Fault Detection, Isolation, and Accommodation Control in Robotic Systems," IEEE Transections on Automation and Engineering, Vol. 5, No. 3, July 2008, pp. 480-489.

[28] X. Zhang, L.F. Ramos, K. Rattan, "Adaptive Fault-Tolerant Fuzzy Control of Robotic Systems," The 28th North American Fuzzy Information Processing Society Annual Conference, Ohio, USA, June 14, 2009.

[29] G. Tao, S. Chen, S.M. Joshi, "An Adaptive Actuator Failure Compensation Controller Using Output Feedback," IEEE Transections on Automatic Control, Vol. 47, No. 3, March 2002, pp. 506-511. 
[30] C. Li, "Adaptive and Robust Composite Control of Coordinated Motion of Space Robot System with Prismatic Joint," Proceeding of the $4^{\text {th }}$ World Congress on Intelligent Control and Automation. Shanghai, China, June 2002.

[31] X. Bai, J.D. Turner, J.L. Junkins, "AIAA Astrodynamics Specialist Conference and Exhibit," Keystone, Colorado, August 2006.

[32] J. Guo, G. Tao, Y. Liu, "A multivariable MRAC scheme with application to a nonlinear aircraft model," Automatica, Vol. 47, 2011, pp. 804-812.

[33] Y. Liu, G. Tao, S.M. Joshi, "Modeling and Model Reference Adaptive Control of Aircraft with Asymmetric Damage," Journal of Guidance Control and Dynamics, Vol. 33, No. 5, September 2010.

[34] A.A. Maciejewski, "Fault Tolerant Properties of Kinematically Redundant Manipulators" IEEE Conference on Robotics and Automation, pp. 638-642, Cincinnati, OH, May 1990.

[35] S.T. Mondschein, G. Tao, "Adaptive Actuator Nonlinearity Compensation and Disturbance Rejection with an Aircraft Application," American Control Conference, San Francisco, CA, USA, June 2011.

[36] G. Liu, "Control of Robot Manipulators with Consideration of Actuator Performance Degradation and Failures," Proceedings of the 2001 IEEE International Conference on Robotics, Seoul, Korea . May 2001.

[37] H. Xu, M. Mirmirani, "Robust Adaptive Sliding Control for A Class of MIMO Nonlinear System," Proceedings of the 2001 AIAA Guidance, Navigation, and Control Conference, Canada, August 2002. 
[38] C. Bonivento, L. Gentili, A. Paoli, "Internal model based fault tolerant control of a robot manipulator," 43rd IEEE Conference on Decision and Control, Atlantis, Paradise Island, Bahamas, December 2004.

[39] A.H. Al-Bayati, H. Wang, "A Robust New Nonlinear Fault Tolerant Control for Robot via an Observer," Proceedings of the 8th World Congress on Intelligent Control and Automation, Taipei, Taiwan, June 2011.

[40] Q. Song, W.J. Hu, L. Yin, Y.C. Soh, "Robust Adaptive Dead Zone Technology for Fault-Tolerant Control of Robot Manipulators Using Neural Networks," Journal of Intelligent and Robotic Systems, Vol. 3, pp. 113-137, 2002.

[41] G. Tao, "Adaptive Control Design and Analysis," John Wiley \& Sons, New York, USA, 2003.

[42] A.G. Siqueira, M.H. Terra, M. Bergerman, "Robust Control of Robots Fault Tolerant Approaches," Springer, London, 2011.

[43] G. Tao, S. Chen, X. Tang, S.M. Joshi, "Adaptive Control of Systems with Actuator Failures," Springer, London, 2004.

[44] H.K. Khalil, "Nonlinear System Third Edition", Printice Hall, New Jersey, USA, 2002.

[45] A.C. Huang, M.C. Chien, "Adaptive Control of Robot Manipulators A Unified Regressor-Free Approach," World Scientific, USA, 2010.

[46] B.C. Kuo, "Digital Control Systems Second Edition," Oxford University Press, New York, 1992.

[47] C.T. Chen, "Linear System Theory and Design," Oxford University Press, New York, 1999. 
[48] S. Cetinkunt, "Mechatronics," John Wiley \& Sons, New York, USA, 2007.

[49] G.F. Franklin, J.D. Powell, A.E. Naeini, "Feedback Control of Dynamic System," Pearson Higher Education, New Jersey, USA, 2010. 